\title{
Cooperative power control in OFDMA small cell networks
}

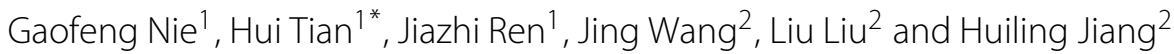

\begin{abstract}
This paper aims at improving the system throughput of orthogonal frequency division multiple access small cell networks. Different with traditional schemes that neglect the cooperation among small cells, a scheme named as resource block exclusion-based power control (RBEBPC) is proposed by sharing the interference correlated information. RBEBPC consists of two steps that are iteratively conducted. First, based on current power allocation results, partial system resource blocks are excluded by playing the formulated cooperative coalition formation games. Second, the transmission power of each small cell is determined by solving a modified throughput maximization problem after the resource block exclusion. As the generated interference is constrained in the second step, part of the small cells transmit without full power. Thereby, the overall system interference keeps non-increasing after RBEBPC is adopted. Simulation results indicate that about 15\% system throughput gain and 13\% power saving gain are obtained compared to traditional iterative water filling scheme.
\end{abstract}

Keywords: Cooperative power control; Orthogonal frequency division multiple access; Small cell; Coalition game; Iterative water filling

\section{Introduction}

The wireless data traffic of cellular system is soaring. Based on the report of future mobile data forecast by Cisco, the compound annual growth rate of mobile data traffic from 2011 to 2016 will be $78 \%$ [1]. The wireless industry faces a big challenge: a 1,000-fold data traffic increase in a decade [2]. The emergence of small cell releases the cost pressure on the dense cell deployment, which makes it one of the most promising solutions to the explosive wireless traffic growth. Small cell is the node whose transmission power is lower than that of the macro base station [3]. Both picocell and femtocell are included in the concept of small cell. Small cell is cheap, plug and play, and self-configuring, which can be easily embedded in the existing cell deployment. The deployment of small cell shortens the distance between service providers and subscribers. As a consequence, subscribers can achieve the same quality of service with lower power consumption. The shorter distance also enables the small cell to

\footnotetext{
*Correspondence: tianhui@bupt.edu.cn

1 State Key Laboratory of Networking and Switching Technology, Beijing University of Posts and Telecommunications, Xitucheng Road, 100876 Beijing, China

Full list of author information is available at the end of the article
}

use a higher carrier frequency that is not suitable for the macro cell.

However, when small cells share the same spectrum band with macro cells, strong interference may exist. There are two categories of interference in small cell networks. The first category, which is known as cross-tier interference, is the interference between small cells and macro cells. The second category, which is known as cotier interference, is the interference among small cells. Strong interference drops the advantages of small cell deployment and becomes the bottleneck of throughput improvement. Some previous works on small cell interference mitigation focus on orthogonal resource reuse from time or frequency domain. Authors of [4-7] propose orthogonal time domain interference mitigation schemes, e.g., almost blank subframe and the enhanced versions. Authors of $[8,9]$ provide frequency domain interference mitigation scheme, e.g., fractional frequency reuse. The orthogonal resource reuse schemes have the common drawback that the interference is mitigated at the cost of resource utilization. Authors of [10] analyze the development trend of small cell networks and point out that small cell deployment would become denser in the future. Marginal reward would be obtained in the orthogonal

\section{照 Springer}

C 2015 Nie et al: licensee Springer. This is an Open Access article distributed under the terms of the Creative Commons

Attribution License (http://creativecommons.org/licenses/by/4.0), which permits unrestricted use, distribution, and reproduction in any medium, provided the original work is properly credited. 
resource reuse schemes due to the large quantity of weak interference sources. In addition to orthogonal resource reuse, power control is also considered to mitigate the interference. Authors of [11] provide a price-based power control scheme to manage the first category of interference. But only one sub-channel case is considered. In [12], hierarchical competition schemes for downlink power allocation in orthogonal frequency division multiple access (OFDMA) femtocell networks are proposed. A Stackelberg game model is used to manage the crosstier interference of Femtocell and macro cell. In [13], the potential game is used with a designed payoff function to manage both the co-tier and cross-tier interferences. Some works adopt the centralized manner to manage the power control issues in small cell networks. Authors of [14] provide centralized suboptimal algorithms to manage the downlink scheduling and power allocation issues in multicell OFDMA networks. Authors of [15] provide a binary power allocation scheme to mitigate the second category of interference but the research is limited to indoor scenario.

As described in [3], the scenario where spectrum bands used for small cells and macro cells are different is one of the scenarios remaining to be studied. Besides, the deployment of small cells are more random and flexible than conventional macro cells, which makes the interference mitigation issues among small cells rather challenging. In this paper, we resort to the cooperative game theory and convex optimization theory to deal with the interference issues in small cell networks. Our target is to establish an effective method to improve the system throughput. Considering the deployment trend $[2,3]$, we only deal with the co-tier interference of the small cells. Based on the resource structure of OFDMA small cell networks, the proposed scheme, which is called resource block exclusion based power control (RBEBPC), conducts the following two steps iteratively. 1) Small cells cooperatively exclude the disadvantage resource blocks by using the coalition formation game theory. An interference constraint is obtained based on the channel exclusion results for each small cell. 2) The transmission power of small cell is determined by solving the modified throughput maximization problems. The system total interference is non-increasing during the conduction of RBEBPC. Besides, RBEBPC is also energy efficient, i.e., not all available power is used during the transmission.

\section{System model and basic assumptions}

In this paper, bold lower case letters denote vectors and bold upper case letters denote matrices. The element of the $i$-th row and the $j$-th column of $\mathbf{X}$ is denoted by $[\mathbf{X}]_{i j}$. The transpose of $\mathbf{X}$ is $\mathbf{X}^{T} \cdot \mathbb{R}^{M \times N}$ represents the $M \times N$ real space and $\mathbb{R}$ represents the real line. Calligraphic upper case letters denote sets. The cardinal number of $\mathcal{S}$ is denoted by $|\mathcal{S}|$, and the power set of $\mathcal{S}$ is denoted by $\eta(\mathcal{S})$. Notation $\left(\begin{array}{l}n \\ k\end{array}\right)$ represents 'n choose k' and equals $\frac{n !}{k !(n-k) !}$. Notation $\Longleftrightarrow$ means 'if and only if'. Finally, 's.t.' is the abbreviation of 'subject to'.

As shown in Figure 1, the considered small cell network is under the coverage of a macro cell. The frequency bandwidth used by the macro cell is F1. The frequency bandwidth shared by small cells is F2. The used spectrum of F1 is orthogonal to that of F2, i.e., there is no cross-tier interference between the macro cell and small cells. Each small cell is assumed to have a backhaul link to the macro cell so that some mutual information can be exchanged. Small cells in the considered system model are denoted by $\mathcal{N}=\{1,2, \ldots, N\}$. Without loss of generality, each small cell is assumed to serve only one user equipment (UE). In other words, in our system, each small cell first schedules a UE, then performs power allocation. The set of UEs is denoted by $\mathcal{M}=\{1,2, \ldots, M\}$ and $M=N$. The $i$-th UE is assumed to be served by the $i$-th small cell. F2 equals $W$ and is equally divided into $L$ sub-channels. The available sub-channels for small cells in the system are denoted by $\mathcal{L}=\{1,2, \ldots, L\}$.

Figure 2 shows the resource structure of the small cell network that is based on the above description. The system resource can be depicted from two dimensions: the small cell dimension and the frequency dimension. In the small cell dimension, each small cell owns at most $L$ available sub-channels and all these sub-channels are able to share the total transmission power $P 0$. However, in the frequency dimension, each sub-channel can be occupied by at most $N$ small cells and these small cells cannot share the transmission power. The resource block of the $l$-th subchannel occupied by the $j$-th small cell (denoted by $R B_{l j}$ ) can be uniquely determined by the tuple $(l, j)$.

The wireless channel is assumed to vary in a slower pace compared to our power control scheme, i.e., the channel

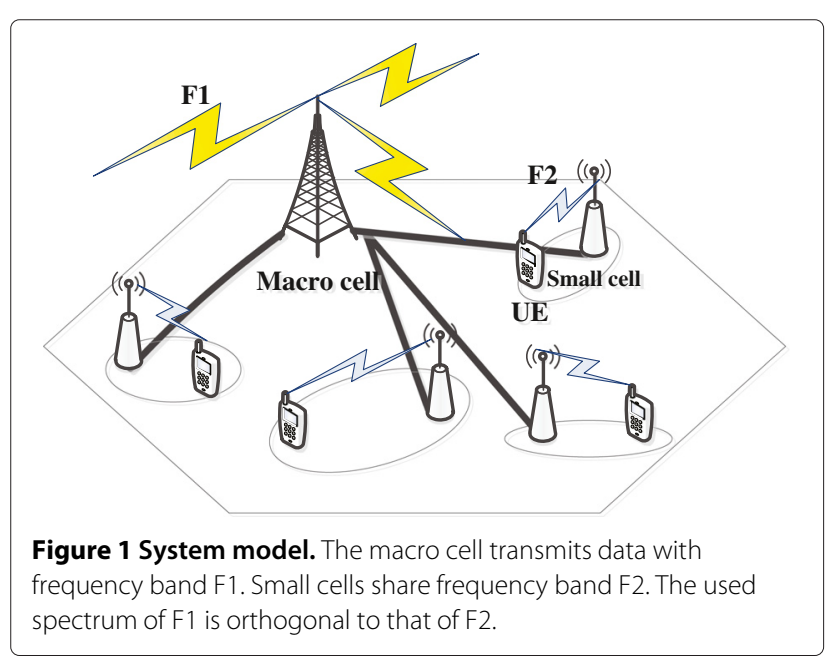




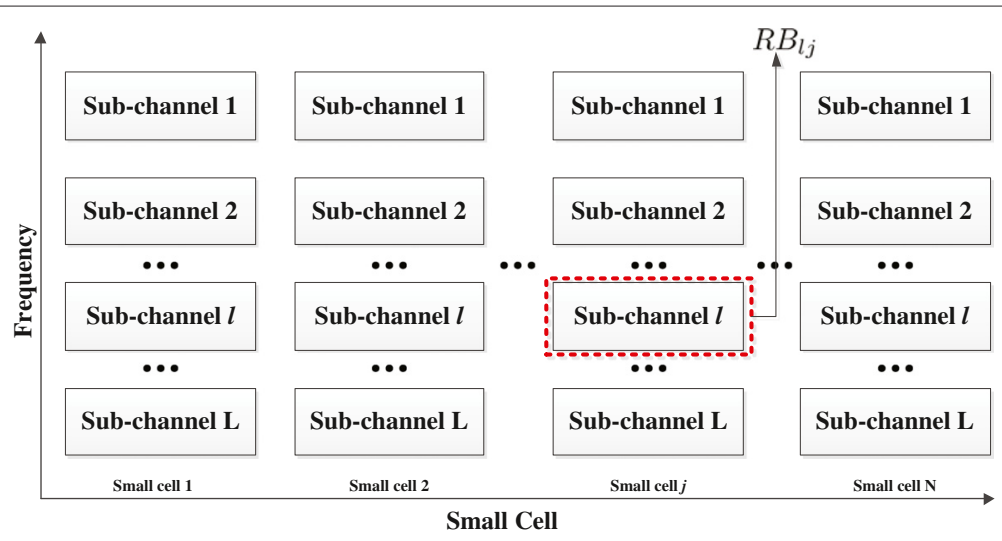

Figure 2 Resource structure of the small cell network.

gain during our analysis remains constant. The channel gain between the $i$-th UE and the $j$-th small cell on the $l$-th sub-channel is denoted by $G_{i j}^{l}$. The system channel gain matrix is denoted by $\mathbf{G} \in \mathbb{R}^{M \times N \times L}$. The channel gain matrix related to the $i$-th $\mathrm{UE}$ is defined as $\mathbf{G}_{i}$. $\in \mathbb{R}^{N \times L}$, which contains all the channel gain between the $i$-th UE and all the small cells on all the sub-channels. Similarly, the channel gain related to the $j$-th small cell and the $l$-th sub-channel are defined as $\mathbf{G}_{. j} \in \mathbb{R}^{M \times L}$ and $\mathbf{G}_{. .}^{l} \in \mathbb{R}^{M \times N}$ separately.

For the $i$-th small cell, the power allocation result is denoted by $\mathbf{p}_{i}=\left[p_{i}^{1}, p_{i}^{2}, \ldots, p_{i}^{L}\right]^{T} \in \mathbb{R}^{L \times 1}$, where $p_{i}^{l}$ represents the allocated power level on the $l$-th sub-channel. The system power allocation result is denoted by $\mathbf{P}=$ $\left[\mathbf{p}_{1}, \mathbf{p}_{2}, \ldots, \mathbf{p}_{N}\right] \in \mathbb{R}^{L \times N}$.

Based on above assumptions, the throughput of the $j$-th small cell is as follows:

$$
R_{j}=\frac{W}{L} \sum_{l=1}^{L} \log _{2}\left(1+\frac{p_{j}^{l} G_{j j}^{l}}{\sigma^{2}+I_{j}^{l}}\right),
$$

where $\sigma^{2}$ is the noise power of each sub-channel and

$$
I_{j}^{l}=\sum_{i=1, i \neq j}^{N} p_{i}^{l} G_{j i}^{l}
$$

is the suffered interference on the $l$-th sub-channel. The transmission power of each small cell is usually constrained by:

$$
\sum_{l=1}^{L} p_{j}^{l} \leq P 0, \forall j \in \mathcal{N},
$$

where $P 0$ is the maximum transmission power of each small cell and

$$
p_{j}^{l} \geq 0, \forall j \in \mathcal{N}, \forall l \in \mathcal{L}
$$

The system throughput maximization problem of the small cell network is described as:

$$
\text { P1 : } \max \sum_{j=1}^{N} R_{j}
$$

s.t. (Equation 3), (Equation 4).

\section{Motivation of cooperative power control}

Since $\mathbf{P 1}$ is non-concave to its domain, it is difficult to obtain the global optimal solution. Some sub-optimal solutions concentrate on the non-cooperative manner such as the iterative water filling (IWF) to slove P1. Due to the neglect of possible mutual cooperation, the noncooperative sub-optimal solutions to P1 may not be efficient. To see this, a simple example of small cell network that motivates the cooperation is analyzed below.

Let us consider a simple small cell network with parameters $W=2, L=2, P 0=1, \mathcal{N}=\{1,2\}, \mathcal{M}=\{1,2\}$, $\sigma^{2}=0.01$. The performance of IWF and equal power allocation (EPA) is discussed. The IWF scheme, which provides a non-cooperative sub-optimal solution to P1, can be perfectly represented as a non-cooperative game $\mathcal{O}=\{\mathcal{N}, \mathcal{Y}, \mathcal{R}\} . \mathcal{N}$ is the player set. $\mathcal{Y}$ is the strategy space whose elements are system power allocation results. $\mathcal{R}$ is the payoff set, in which the $j$-th $(\forall j \in \mathcal{N})$ element is $R_{j}$. In IWF, the $j$-th small cell selfishly determines $\mathbf{p}_{j}$ to maximize $R_{j}$ based on previous interference level. By conducting the power allocation for several rounds, the system can reach a stable state where each small cell has no incentive to change its power allocation results. The EPA scheme just equally allocates the available power to all sub-channels. Two scenarios with above system parameters are considered. Scenario 1 is a strong interference scenario, whose system channel matrix $\mathbf{G} \in \mathbb{R}^{2 \times 2 \times 2}$ is described as:

$$
\mathbf{G}_{. .}^{1}=\left[\begin{array}{ll}
0.7 & 0.3 \\
0.3 & 0.5
\end{array}\right], \mathbf{G}_{. .}^{2}=\left[\begin{array}{ll}
0.9 & 0.5 \\
0.5 & 0.8
\end{array}\right]
$$


Scenario 2 is a weak interference scenario whose system channel matrix $\mathbf{F} \in \mathbb{R}^{2 \times 2 \times 2}$ is as follows:

$$
\mathbf{F}_{. .}^{1}=\left[\begin{array}{cc}
0.7 & \mathbf{0 . 0 3} \\
\mathbf{0 . 0 3} & 0.5
\end{array}\right], \mathbf{F}_{. .}^{2}=\left[\begin{array}{cc}
0.9 & \mathbf{0 . 0 5} \\
\mathbf{0 . 0 5} & 0.8
\end{array}\right] \text {. }
$$

The system throughput of IWF with above system configuration is shown in Figure 3. The start point of each sub-figure is the system throughput of EPA. We can see that the system throughput improves about $8 \%$ at the stable state in scenario 1 . But in scenario 2 , the throughput improvement of IWF can be neglected.

However, if small cells in scenario 1 follow some cooperation rules, the following power allocation can be obtained:

$$
\hat{\mathbf{P}}=\left[\begin{array}{ll}
p_{1}^{1} & p_{2}^{1} \\
p_{1}^{2} & p_{2}^{2}
\end{array}\right]=\left[\begin{array}{ll}
1 & 0 \\
0 & 1
\end{array}\right]
$$

and the corresponding throughput is $\left(R_{1}, R_{2}\right)=$ $(6.5850,5.9307)$. Since both of the small cells improve the throughput by more than $70 \%$ compared to the stable state of IWF, $\hat{\mathbf{P}}$ is more favored. If the total transmission power is further reduced by $20 \%$, the following solution is achieved:

$$
\tilde{\mathbf{P}}=\left[\begin{array}{ll}
p_{1}^{1} & p_{2}^{1} \\
p_{1}^{2} & p_{2}^{2}
\end{array}\right]=\left[\begin{array}{cc}
0.8 & 0 \\
0 & 0.8
\end{array}\right]
$$

and the corresponding throughput is $\left(R_{1}, R_{2}\right)=$ $(6.2668,5.6147)$, which is comparable to the full transmission power allocation results $\hat{\mathbf{P}}$. The great improvements of $\hat{\mathbf{P}}$ and $\tilde{\mathbf{P}}$ motivate the cooperation of small cell networks where strong interference exists. Besides, for the non-cooperative solutions such as IWF, the convergence is not always guaranteed [16].

In this paper, we aim at achieving such cooperation gain. Some papers seek cooperation solution to the interference issues in small cells via coalition game. Authors of [17] use the recursive core approach to cluster the small cells.
Small cells first form clusters by using the recursive core approach. To mitigate the interference, a time division duplex scheduling strategy is used in the formed clusters. The scheme proposed in [18] promotes the scheme in [17] by allowing a small cell to join more than one coalitions. There are obvious differences between the schemes in $[17,18]$, and RBEBPC which will be introduced in the next section. First, both the schemes proposed in $[17,18]$ are distributed schemes, while RBEBPC is a centralized solution. Second, no power control is used in the schemes of $[17,18]$, while RBEBPC uses the power control method to deal with the interference.

\section{Resource block exclusion-based power control}

In RBEBPC, the goal is to improve the system throughput by excluding the strong interference resource blocks and by redistributing the system interference among small cells. To conduct RBEBPC, some mutual interference correlated information, such as $\mathbf{G}$ and previous $\mathbf{P}$, should be exchanged among small cells. Considering the large number of small cells and the backhaul ability of each small cell, the centralized cooperation structure is adopted to guarantee the efficiency of information exchange. The macro cell, which covers all the small cells in the system, acts as the centralized node to collect and forward all the necessary information. The procedure of RBEBPC is described in Algorithm 1.

In order to obtain $\mathbf{G}$, in the initialization state, all small cells transmit with equal power on all available sub-channels (line 3 in Algorithm 1). Then, the macro cell collects and forwards the necessary part of $\mathbf{G}$ to the small cells (line 4 and line 5). After information collection, small cells will conduct the cooperative power control for $K$ rounds. In each round, small cells first report the used power levels to the macro cell (line 7). The macro cell, based on the reported power level, plays the
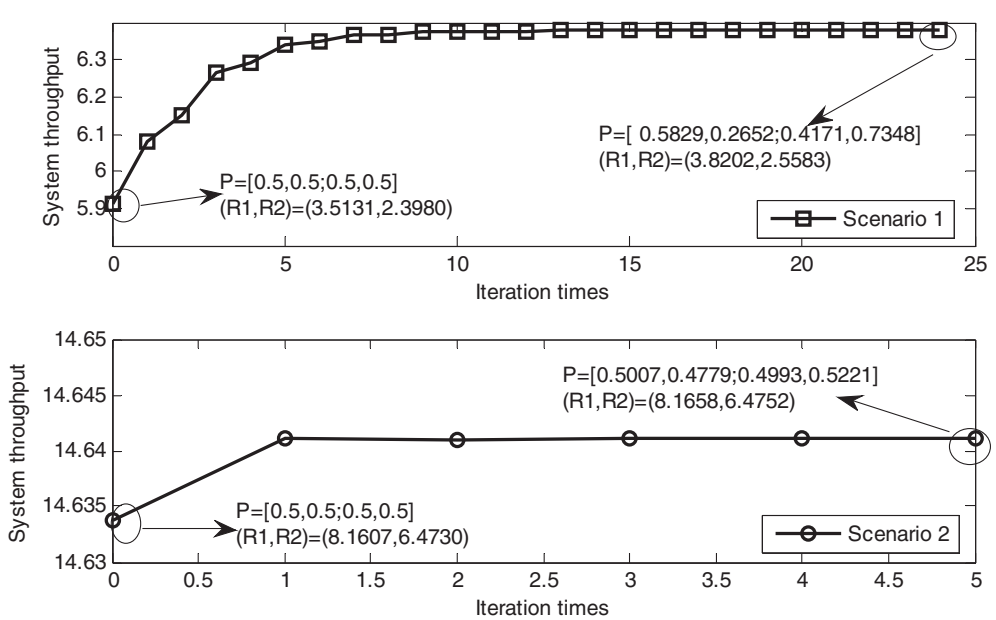

Figure 3 System throughput of IWF. 
resource block exclusion game and calculates the interference constraint based on the resource block exclusion results for each small cell (step 1). Then each small cell solves a throughput maximization problem by following the received constraint from the macro cell (step 2).

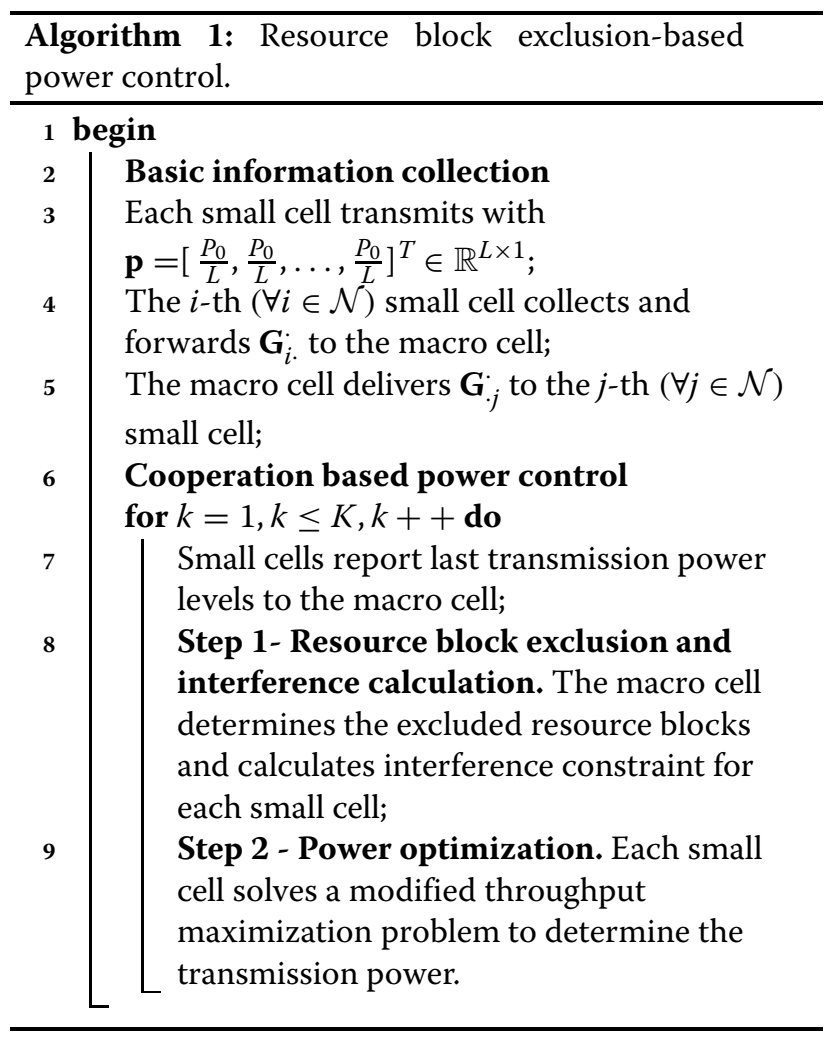

The necessary signaling overhead among the small cells, the serving UEs, and the macro cell in RBEBPC scheme can be estimated as follows. In the Basic information collection phase, if the quantification of a sub-channel gain of a certain small cell needs $\alpha$ bits, each UE should feedback $\alpha N L$ bits to the access small cell to indicate the interfering channel gain on all the sub-channels. Then, each small cell forwards the $\alpha N L$ bits to the macro cell via the backhaul link. Each small cell receives $\alpha N L$ bits information to indicate the channel gains correlated to the interfering UEs in the system. In the Cooperation based power control phase, each small cell forwards $\beta L$ bits to indicate the previous power level in step 1 , where $\beta$ is the necessary bits to quantify the power level on a sub-channel. Each small cell receives $N L$ bits that indicate the resource block exclusion results and receives $\gamma(L+1)$ bits that indicate the suffered interference on each sub-channel and the generated total interference, where $\gamma$ is the necessary bits to quantify the interference level on a sub-channel. So in Algorithm 1, the signaling overhead between a UE and the serving small cell is $\alpha N L$ bits. The signaling overhead between a small cell and the macro cell is $2 \alpha N L+K(\beta L+N L+\gamma(L+1))$ bits. As for the typical wireless channel, the time scale for change of path amplitude is several hundreds of millisecond [19]. In long-term evolution (LTE), the transmission time interval is $1 \mathrm{~ms}$, which is much smaller than the time of path amplitude change. So in the extreme case, the backhaul link between the small cell and the macro cell should satisfy the data rate $\max \{\alpha N L, \beta L, N L+\gamma(L+1)\}$ kbps. For the typical configuration $\alpha=\beta=\gamma=64$, $N=10, L=50$, the backhaul link data rate should satisfy $0.032 \mathrm{Gbps}$. If the backhaul links are implemented by using the standardized passive optical network (PON) [20] systems whose downstream is about $2.5 \mathrm{Gbps}$, the signaling overhead of RBEBPC is acceptable in practice.

In the following subsections, the resource block exclusion, interference calculation, and power optimization in RBEBPC are described in detail.

\section{Resource block exclusion}

Given the system power allocation result $\mathbf{P}$ and system channel gain matrix $\mathbf{G}$, a binary matrix $\mathbf{A} \in\{0,1\}^{L \times N}$ is defined to map the available sub-channels in the system:

$$
[\mathbf{A}]_{l j}=\left\{\begin{array}{l}
1, \text { if }[\mathbf{P}]_{l j}>0 \\
0, \text { if }[\mathbf{P}]_{l j} \leq 0
\end{array}\right.
$$

For the $j$-th small cell, the $l$-th sub-channel is available if and only if $[\mathbf{A}]_{l j}=1$. Otherwise, $[\mathbf{A}]_{l j}=0$ and the $j$-th small cell does not use the $l$-th sub-channel.

In Figure 2, the total available system resource blocks can be partitioned into $\mathcal{C}=\left\{\mathcal{N}_{1}, \mathcal{N}_{2}, \ldots, \mathcal{N}_{L}\right\}$, where $|\mathcal{C}|=$ $L$ and $\mathcal{N}_{l} \subseteq \mathcal{N}$ denotes the set of small cells for which the $l$-th sub-channel is available (i.e., $\mathcal{N}_{l}=\left\{j \in \mathcal{N} \mid[\mathbf{A}]_{l j}=1\right\}$ ). Given a sub-channel, the allocated power is not transferable, i.e., the power cannot be shared among small cells in an arbitrary ratio. For the sub-channels with strong interference, the system throughput will improve if some small cells mute the sub-channels. In this subsection, we resort to the coalition game to determine the sub-channels that need to be muted. Note that the system interference on the $l$-th sub-channel is only determined by the small cells in $\mathcal{N}_{l}$. Besides, the system interferences of different sub-channels are independent once $\mathbf{P}$ is given. So in the following analysis, we only consider the $l$-th sub-channel and the correlated small cells in $\mathcal{N}_{l}$.

Definition 1. Let $\mathcal{N}=\{1,2, \ldots, N\}$ be a set of fixed players called the grand coalition. Non-empty subsets of $\mathcal{N}$ are called coalitions. A collection (in the grand coalition $\mathcal{N})$ is any family $\mathcal{D}=\left\{\mathcal{D}_{1}, \mathcal{D}_{2}, \ldots, \mathcal{D}_{s}\right\}$ of mutually disjoint coalition, and $s$ is called its size. If additionally $\cup_{t=1}^{s} \mathcal{D}_{t}=\mathcal{N}$, the collection $\mathcal{D}$ is called a partition of $\mathcal{N}$.

Based on Definition 1, the throughput of a coalition $\mathcal{E} \subseteq$ $\mathcal{N}_{l}$ is as follows: 


$$
R(\mathcal{E})=\sum_{j \in \mathcal{E}} \frac{W}{L} \log _{2}\left(1+\frac{p_{j}^{l} G_{j j}^{l}}{\sigma^{2}+I_{j}^{l}}\right) .
$$

If the small cells in the coalition $\mathcal{E}$ mute in a cooperative manner and only one small cell in $\mathcal{E}$ transmits, the achievable throughput is as follows:

$$
\tilde{R}(\mathcal{E})=\max _{j \in \mathcal{E}} \frac{W}{L} \log _{2}\left(1+\frac{p_{j}^{l} G_{j j}^{l}}{\sigma^{2}+\sum_{i \in \mathcal{N}_{l} \backslash \mathcal{E}} p_{i}^{l} G_{j i}^{l}}\right) .
$$

The formulated coalition game of the $l$-th sub-channel is denoted by $\mathcal{G}^{l}=\left(\mathcal{N}_{l}, v\right)$, where $\mathcal{N}_{l}$ is the set of players and $v: \eta\left(\mathcal{N}_{l}\right) \mapsto \mathbb{R}$ is the payoff function of the coalitions [21]. The payoff function should take the interference and system throughput into consideration. A suitable payoff function for coalition $\mathcal{E}\left(\forall \mathcal{E} \in \eta\left(\mathcal{N}_{l}\right)\right)$ is as follows:

$$
v(\mathcal{E})= \begin{cases}\tilde{R}(\mathcal{E}), & \text { if } R(\mathcal{E})<\tilde{R}(\mathcal{E}), \\ 0, & \text { if } R(\mathcal{E}) \geq \tilde{R}(\mathcal{E}) .\end{cases}
$$

Note that for $\emptyset$, we have $v(\emptyset)=0$. Based on Equation 13, the establishment of coalition $\mathcal{E}(|\mathcal{E}| \geq 2)$ has two effects. The first effect is the reduction of transmission resource blocks for small cells in $\mathcal{E}$. Before the formulation of $\mathcal{E}$, small cells in $\mathcal{E}$ can have at most $|\mathcal{E}|$ transmission resource blocks (i.e., each small cell owns a transmission resource block) and at least two transmission resource blocks (i.e., $\mathcal{E}$ is established based on two existing coalitions). However, when $\mathcal{E}$ is formulated, small cells in $\mathcal{E}$ only have one transmission resource block. The second effect is the reduction of interference of the remaining transmission resource block. Since the small cells in $\mathcal{E}$ mute in a cooperative manner, the interference of small cells in $\mathcal{E}$ is canceled.

Some properties of $\mathcal{G}^{l}$ are summarized as follows.

Definition 2. The form of a coalition game $(\mathcal{N}, v)$ is the characteristic form, if and only if the value of a coalition $\mathcal{E} \in \eta(\mathcal{N})$ solely depends on the players in $\mathcal{E}$ and is in independent with how the players in $\mathcal{N} \backslash \mathcal{E}$ are structured.

Property 1. The form of game $\mathcal{G}^{l}$ is the characteristic form.

Proof. This property directly follows by the definition of payoff function (Equation 13).

From Property 1, we can see that the value of the formulated payoff function is only sensitive to the players in the given coalition. In small cell networks, small cells are coupled with each other via the effect of interference. In (Equation 13), the effect of small cells beyond the given coalition is treated as a whole and is independent with their partition structure. By using Equation 13, we can obtain the value of a coalition once the coalition is given.
Given a partition $\mathcal{P}=\left\{\mathcal{P}_{1}, \mathcal{P}_{2}, \ldots, \mathcal{P}_{s}\right\}$ of $\mathcal{N}_{l}$, we can find a unique vector $\mathbf{v}=\left[v\left(\mathcal{P}_{1}\right), v\left(\mathcal{P}_{2}\right), \ldots, v\left(\mathcal{P}_{s}\right)\right]^{T} \in \mathbb{R}^{s \times 1}$ to represent the value of each coalition in $\mathcal{P}$. Besides, since the coalition value of $\mathcal{G}^{l}$ is the maximum achievable throughput by a single member small cell, game $\mathcal{G}^{l}$ has a transferable utility, i.e., the achievable throughput can be arbitrarily portioned among the small cells of a coalition (for example, via the proper choice of coding strategy [18]).

Definition 3. A transferable utility coalition game is supperadditive if and only if $\forall \mathcal{E}_{1} \subseteq \mathcal{N}_{l}, \mathcal{E}_{2} \subseteq \mathcal{N}_{l}$ and $\mathcal{E}_{1} \cap \mathcal{E}_{2}=\emptyset$ :

$$
v\left(\mathcal{E}_{1} \cup \mathcal{E}_{2}\right) \geq v\left(\mathcal{E}_{1}\right)+v\left(\mathcal{E}_{2}\right) .
$$

For the transferable utility coalition game with supperadditivity, establishing a bigger coalition is always beneficial.

Property 2. Due to the implied tradeoff between interference and throughput in (Equation 13), the formulated game $\mathcal{G}^{l}$ is not supperadditive and the grand coalition is not always formed; thus, disjoint independent coalitions will form in the network.

The proof of Property 2 can be found in the Appendix. The payoff function (Equation 13) shows that in the formulated coalition, only one player transmits and the rest in the coalition keep silent. Once a coalition is formulated, the available number of resource blocks reduces but the quality of the remaining resource block improves. Due to the random deployment of small cell and the fluctuation of the wireless channel quality, small cells may not be prone to form the grand coalition. In small-scale area where strong interference exists, small cells form a coalition with a higher possibility since the payoff of the coalition is probably higher if the interference is removed. The main obstacle of the grand coalition construction lies in (Equation 13) because the reduction of the available resource blocks is the cost of coalition construction. In practice, the construction of grand coalition needs harsh conditions. In the case that the interferences among small cells are so strong that if more than one small cell share the sub-channel the total throughput of the sub-channel reduces, the grand coalition will form.

Definition 4. A comparison relation $\triangleright$ is defined for two collections $\mathcal{D}=\left\{\mathcal{D}_{1}, \mathcal{D}_{2}, \ldots, \mathcal{D}_{s}\right\}$ and $\mathcal{F}=$ $\left\{\mathcal{F}_{1}, \mathcal{F}_{2}, \ldots, \mathcal{F}_{w}\right\}$ that satisfy $\bigcup_{m=1}^{s} \mathcal{D}_{m}=\bigcup_{n=1}^{w} \mathcal{F}_{n}=\mathcal{H} \subseteq$ $\mathcal{N}$. Thus, $\mathcal{D} \triangleright \mathcal{F}$ means that the way $\mathcal{D}$ partitions $\mathcal{H}$ is preferred to the way $\mathcal{F}$ partitions $\mathcal{H}$.

Definition 5. For collection $\mathcal{D}$ and $\mathcal{F}$ defined in Definition 4: 


$$
\mathcal{D} \triangleright \mathcal{F} \Longleftrightarrow \sum_{m=1}^{s} v\left(\mathcal{D}_{m}\right)>\sum_{n=1}^{w} v\left(\mathcal{F}_{n}\right) \text {. }
$$

Definitions 4 and 5 provide a preference among partitions. Definition 5 indicates that 'social welfare' (the total throughput) is considered as the baseline. So the defined preference is coordinate with the target of system throughput improvement. We can use the exhaustive search method to obtain the maximum throughput partition structure of $\mathcal{G}^{l}$, in which a rather large search space should be considered. For the player set $\mathcal{N}_{l}$, the number of possible partition, which is given by a value known as Bell number, grows sharply with the number of players in $\mathcal{N}_{l}$. For example, the Bell number of $\mathcal{N}_{l}$ is 115,975 when $\left|\mathcal{N}_{l}\right|=10$. However, by following some simple rules, we can obtain a stable practical partition structure of $\mathcal{G}^{l}$.

For any two collections $\mathcal{D}=\left\{\mathcal{D}_{1}, \mathcal{D}_{2}, \ldots, \mathcal{D}_{s}\right\}, \mathcal{F}=$ $\left\{\mathcal{F}_{1}, \mathcal{F}_{2}, \ldots, \mathcal{F}_{w}\right\}$ and the grand coalition $\mathcal{N}$ that satisfy $\left(\bigcup_{m=1}^{s} \mathcal{D}_{m}\right) \cup\left(\cup_{n=1}^{w} \mathcal{F}_{n}\right)=\mathcal{N}$ and $\left(\cup_{m=1}^{s} \mathcal{D}_{m}\right) \cap\left(\cup_{n=1}^{w} \mathcal{F}_{n}\right)=$ $\emptyset$, we define two operation rules to construct the stable partition structure.

Definition 6. Merge rule: If $\left\{\bigcup_{m=1}^{s} \mathcal{D}_{m}\right\} \cup \mathcal{F} \triangleright \mathcal{D} \cup \mathcal{F}$, merge $\mathcal{D}$ into $\left\{\bigcup_{m=1}^{s} \mathcal{D}_{m}\right\}$ :

$$
\begin{aligned}
& \operatorname{Merge}(\mathcal{D} \cup \mathcal{F})= \\
& \left\{\begin{array}{l}
\left\{\bigcup_{m=1}^{s} \mathcal{D}_{m}\right\} \cup \mathcal{F}, \quad \text { if }\left\{\bigcup_{m=1}^{s} \mathcal{D}_{m}\right\} \cup \mathcal{F} \triangleright \mathcal{D} \cup \mathcal{F}, \\
\mathcal{D} \cup \mathcal{F}, \quad \text { if } \mathcal{D} \cup \mathcal{F} \triangleright\left\{\bigcup_{m=1}^{s} \mathcal{D}_{m}\right\} \cup \mathcal{F} .
\end{array}\right.
\end{aligned}
$$

Definition 7. Split rule: If $\mathcal{D} \cup \mathcal{F} \triangleright\left\{\cup_{m=1}^{s} \mathcal{D}_{m}\right\} \cup \mathcal{F}$, split $\left\{\bigcup_{m=1}^{s} \mathcal{D}_{m}\right\}$ into $\mathcal{D}$ :

$$
\begin{aligned}
& \operatorname{Split}\left(\left\{\cup_{m=1}^{s} \mathcal{D}_{m}\right\} \cup \mathcal{F}\right)= \\
& \left\{\begin{array}{l}
\left\{\bigcup_{m=1}^{s} \mathcal{D}_{m}\right\} \cup \mathcal{F}, \quad \text { if }\left\{\bigcup_{m=1}^{s} \mathcal{D}_{m}\right\} \cup \mathcal{F} \triangleright \mathcal{D} \cup \mathcal{F}, \\
\mathcal{D} \cup \mathcal{F}, \quad \text { if } \mathcal{D} \cup \mathcal{F} \triangleright\left\{\bigcup_{m=1}^{s} \mathcal{D}_{m}\right\} \cup \mathcal{F} .
\end{array}\right.
\end{aligned}
$$

Note that the above operation rules of Definitions 6 and 7 use $\triangleright$ 'locally', by focusing on the coalitions that take part and result from the merge operation and split operation. Algorithm 2 summarizes the procedure that uses the merge-split rule to obtain the stable partition structure of $\mathcal{G}^{l}$. Due to Property 1, the conduction of the split rule in Algorithm 2 can be transformed into the merge rule, if the coalition remains to be splitted is treated as a smaller grand coalition. The split operation is equivalent to the merge operation in the smaller grand coalition as long as we treat the effect of the players beyond the smaller grand coalition as a whole.
It is difficult to directly describe the complexity of Algorithm 2, because the termination condition of Algorithm 2 depends on the specific state of the small cell network. But we can estimate the complexity of Algorithm 2 in some extreme cases. Since the split operation can be treated as a special kind of merge operation, we only analyze the complexity of merge operation here. In the worst case where no coalition with size bigger than two is formed, the potential number of possible coalitions that should be considered is $\zeta_{1}=2^{\left|\mathcal{N}_{l}\right|}-$ $\left|\mathcal{N}_{l}\right|-1=\sum_{k=2}^{\left|\mathcal{N}_{l}\right|}\left(\begin{array}{c}\left|\mathcal{N}_{l}\right| \\ k\end{array}\right)$. While in the best case where the grand coalition is formed, the potential number of possible coalitions that should be considered is $\zeta_{2}=\left|\mathcal{N}_{l}\right|-$ 1. So for each merge or split operation, the number of coalitions that should be considered is between $\zeta_{1}$ and $\zeta_{2}$.

Definition 8. A partition $\mathcal{P}=\left\{\mathcal{P}_{1}, \mathcal{P}_{2}, \ldots, \mathcal{P}_{s}\right\}$ of the grand coalition $\mathcal{N}$ is $\mathbb{D}_{c}$ stable, if no players in $\mathcal{N}$ are interested in leaving $\mathcal{P}$ through any operation (not necessary merge or split) to form a partition different with $\mathcal{P}$. A partition $\mathcal{Q}=\left\{\mathcal{Q}_{1}, \mathcal{Q}_{2}, \ldots, \mathcal{Q}_{w}\right\}$ of the grand coalition $\mathcal{N}$ is $\mathbb{D}_{h p}$ stable if no coalition has the incentive to split or merge.

Property 3. The partition structure of $\mathcal{G}^{l}$ obtained by Algorithm 2 is $\mathbb{D}_{h p}$ stable. The existence of $\mathbb{D}_{c}$ stable partition in game $\mathcal{G}^{l}$ is not always guaranteed. If the $\mathbb{D}_{c}$ stable partition of $\mathcal{G}^{l}$ exists, the partition structure obtained by Algorithm 2 is $\mathbb{D}_{c}$ stable.

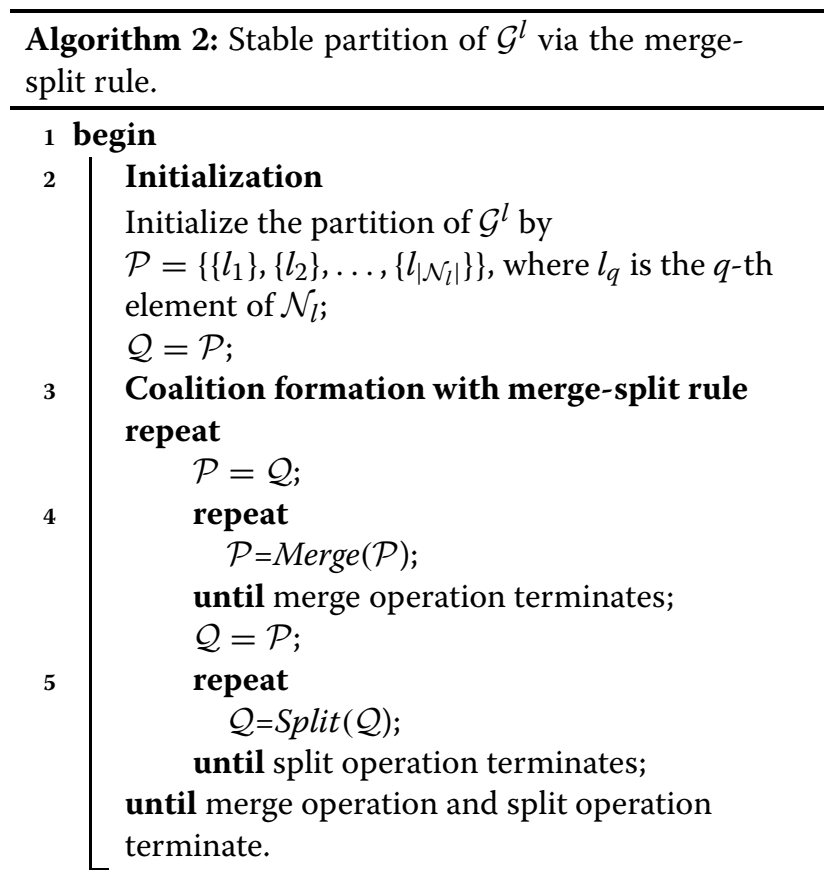


The proof of Property 3 can be found in the Appendix. Property 3 indicates that the final outcome of Algorithm 2 is stable. The outcome of Algorithm 2 can not be improved by any merge or split operation. Generally speaking, the optimal partition results can be found by using the exhaustive method. But the number of possible cases (the Bell number) that should be considered in the exhaustive method is too huge to manage. The outcome of Algorithm 2 may not be globally optimal but it is at least stable.

Denote the outcome of Algorithm 2 by $\mathcal{P}^{*}=$ $\left\{\mathcal{P}_{1}^{*}, \mathcal{P}_{2}^{*}, \ldots, \mathcal{P}_{x}^{*}\right\}$. From Equation 13, we can find the set of players $\mathcal{U}=\left\{y_{1}, y_{2}, \ldots, y_{x}\right\}$, where $y_{t} \in \mathcal{P}_{t}^{*}$ is the player whose throughput on the sub-channel equals $\tilde{R}\left(\mathcal{P}_{t}^{*}\right)$, if other players in $\mathcal{P}_{t}^{*}$ mute in the sub-channel. So the resource blocks with strong interference are marked by modifying the available sub-channel mapping matrix:

$$
[\mathbf{A}]_{l j}=\left\{\begin{array}{l}
1, \text { for } j \in \mathcal{U} \\
0, \text { for } j \in \mathcal{N}_{l} \backslash \mathcal{U}
\end{array}\right.
$$

The resource blocks with strong interference are excluded by setting zero value to the corresponding elements in the available sub-channel mapping matrix. Figure 4 shows a schematic diagram of resource block exclusion. Part of the total system blocks are excluded by playing the formulated games. A detailed example of the resource exclusion procedure can be found in the simulation part.

\section{Interference calculation}

After the resource block exclusion, the suffered interference of the $j$-th UE on the $l$-th sub-channel is as follows:

$$
\tilde{I}_{j}^{l}=\sum_{i=1, i \neq j}^{N} p_{i}^{l} G_{j i}^{l}[\mathbf{A}]_{l i} .
$$

The interference power level on the excluded resource block is not considered in $\tilde{I}_{j}$. The generated interference of small cell $j$ is defined as:

$$
\bar{I}_{j}=\sum_{l=1}^{L} \sum_{i=1, i \neq j}^{M} p_{j}^{l} G_{i j}^{l}[A]_{l i} .
$$

The generated interference depends on three factors: the previous allocated power vector, the interference channel gain and the channel availability. If the subchannel is unavailable for some small cells, the interference to the small cell on this sub-channel has no contribution to the generated interference. Based on the system channel gain matrix G, previous system power allocation results $\mathbf{P}$ and system current mapping matrix A, the macro cell calculates the generated interference vector $\overline{\mathbf{I}}=\left[\bar{I}_{1}, \bar{I}_{2}, \ldots, \bar{I}_{N}\right]^{T} \in \mathbb{R}^{N \times 1}$ and the suffered interference vector $\tilde{\mathbf{I}}_{j}=\left[\tilde{I}_{j}^{1}, \tilde{I}_{j}^{2}, \ldots, \tilde{I}_{j}^{L}\right]^{T} \in \mathbb{R}^{L \times 1}$ $(\forall j \in \mathcal{M})$ after the sub-channel exclusion operation. The two interference correlated vectors are delivered to the small cells to be used in the power optimization procedure.

\section{Power optimization}

After obtaining the interference constraint $\overline{\mathbf{I}}$ and available sub-channel mapping matrix $\mathbf{A}$, each small cell will optimize the transmission power on each available subchannel based on the last transmission power allocation result $\mathbf{P}$. Instead of solving $\mathbf{P 1}$, the $j$-th $(\forall j \in \mathcal{N})$ small cell will solve the following problem:

$$
\begin{array}{ll}
\text { P2: } & \max \sum_{l \in \mathcal{L}_{j}} \frac{W}{L} \log _{2}\left(1+\frac{p_{j}^{l} G_{i j}^{l}}{\sigma^{2}+\tilde{I}_{j}^{l}}\right) \\
\text { s.t. } & \sum_{i \in \mathcal{M} \backslash\{j\}} \sum_{l \in \mathcal{L}_{j}} p_{j}^{l} G_{i j}^{l}[A]_{l i} \leq \bar{I}_{j},
\end{array}
$$

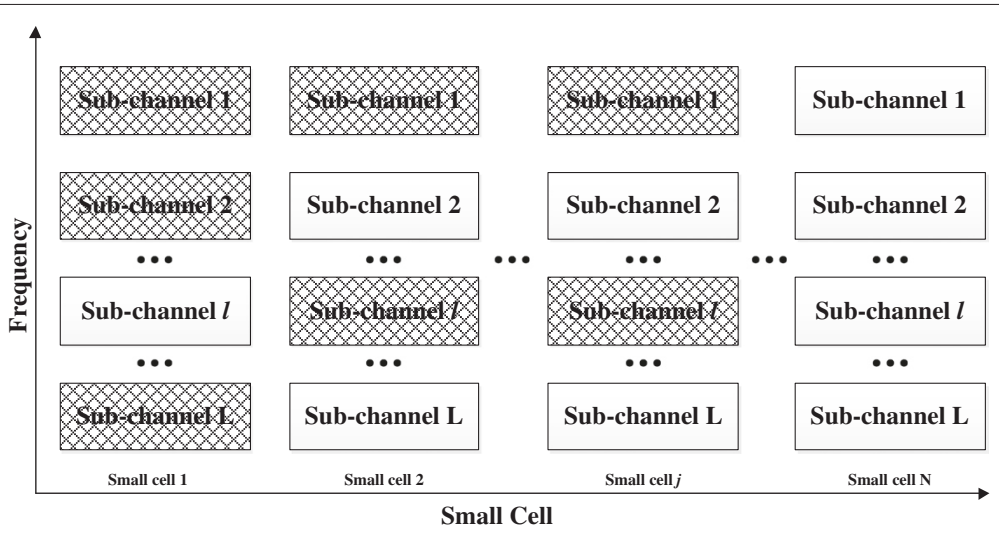

Figure 4 A schematic diagram of resource block exclusion. The grid resource blocks such as $R B_{11}$ and $R B_{12}$ are excluded. From the small cell dimension, each small cell may own more than one sub-channel. From the frequency dimension, each sub-channel can be occupied by more than one small cell. 


$$
\begin{aligned}
& \sum_{l \in \mathcal{L}_{j}} p_{j}^{l} \leq P 0, \\
& p_{j}^{l} \geq 0, \forall l \in \mathcal{L}_{j},
\end{aligned}
$$

where $\mathcal{L}_{j}$ is the set of available sub-channels for the $j$-th small cell (i.e., $\mathcal{L}_{j}=\left\{l \in \mathcal{L} \mid[\mathbf{A}]_{l j}=1\right\}$ ). The constraint of (Equation 22) is used to redistribute the total generated interference on all the available sub-channels. The formulated P2 is a concave problem and the proof can be found in the Appendix.

Since P2 is concave to the domain $\mathbf{p}_{j} \in \mathbb{R}^{\left|\mathcal{L}_{j}\right| \times 1}$, we can use the interior point method [22] to solve it. However, it is more convenient to solve the dual problem of P2, if we take the problem structure into consideration. First, due to concavity, the optimal solution to P2 is equivalent to the optimal solution to the dual problem. Second, the number of variables in P2 is $\left|\mathcal{L}_{j}\right|$. While in the dual problem, the number of variables reduces to two. Because constraints (Equation 24) are treated as slack conditions, only (Equation 22) and (Equation 23) are used when solving the dual problem. Third, in some special cases, the close form solution to the dual problem can be obtained, which greatly accelerates the computation. The details of solving the dual problem are represented as follows. The Lagrangian of P2 is as follows:

$$
\begin{aligned}
& L\left(\lambda, \mu, \theta, \mathbf{p}_{j}\right)= \\
& \sum_{l \in \mathcal{L}_{j}} \frac{W}{L} \log _{2}\left(1+\frac{p_{j}^{l} G_{j j}^{l}}{\sigma^{2}+\tilde{I}_{j}^{l}}\right)+\lambda\left(\bar{I}_{j}-\right. \\
& \left.\sum_{i \in \mathcal{M} \backslash\{j\}} \sum_{l \in \mathcal{L}_{j}} p_{j}^{l} G_{i j}^{l}[A]_{l i}\right)+\mu\left(P 0-\sum_{l \in \mathcal{L}_{j}} p_{j}^{l}\right)+\sum_{l \in \mathcal{L}_{j}} \theta_{l} p_{j}^{l} \\
& \equiv \sum_{l \in \mathcal{L}_{j}}\left[z \log _{2}\left(b_{j}^{l}+p_{j}^{l}\right)+a_{j}^{l}\right]+\lambda\left(\bar{I}_{j}-\sum_{l \in \mathcal{L}_{j}} c_{j}^{l} p_{j}^{l}\right)+ \\
& \mu\left(P 0-\sum_{l \in \mathcal{L}_{j}} p_{j}^{l}\right)+\sum_{l \in \mathcal{L}_{j}} \theta_{l} p_{j}^{l},
\end{aligned}
$$

where $z=\frac{W}{L}, a_{j}^{l}=\frac{W}{L} \log _{2}\left(\frac{G_{j j}^{l}}{\sigma^{2}+\tilde{I}_{j}^{L}}\right), b_{j}^{l}=\frac{\sigma^{2}+\tilde{I}_{j}^{l}}{G_{j j}^{l}}, c_{j}^{l}=$ $\sum_{i \in \mathcal{M} \backslash\{j\}} G_{i j}^{l}[\mathbf{A}]_{l i}$. The first-order partial derivatives of $p_{j}^{l}$ is as follows:

$$
\frac{\partial L\left(\lambda, \mu, \theta, \mathbf{p}_{j}\right)}{\partial p_{j}^{l}}=\frac{z}{\ln 2} \frac{1}{b_{j}^{l}+p_{j}^{l}}-\lambda c_{j}^{l}-\mu+\theta_{l} .
$$

Based on the KKT condition of P2, we can see that $\theta$ is a slack variable vector that can be eliminated [22].
By solving $\frac{\partial L\left(\lambda, \mu, \mathbf{p}_{j}\right)}{\partial p_{j}^{l}}=0$ and substituting the results to Equation 25, we can obtain the dual problem of P2:

$$
\begin{aligned}
& \text { P3: } \min g(\lambda, \mu) \\
& =\min _{\mathbf{p}_{j}} \max _{\mathbf{p}_{j}} L\left(\lambda, \mu, \mathbf{p}_{j}\right) \\
& =\min _{\lambda, \mu} \sum_{l \in \mathcal{L}_{j}}\left[-z \log _{2}\left(\lambda c_{j}^{l}+\mu\right)+\left(\lambda c_{j}^{l}+\mu\right) b_{j}^{l}\right]+ \\
& \lambda \bar{I}_{j}+\mu P 0+\sum_{l \in \mathcal{L}_{j}}\left[z \log _{2}\left(\frac{z}{\ln 2}\right)+a_{j}^{l}-\frac{z}{\ln 2}\right]
\end{aligned}
$$

s.t. $\lambda \geq 0, \mu \geq 0$.

It is easy to verify that $\mathbf{P 3}$ is a convex optimization problem. By using the KKT conditions, constraints (Equation 28) can be neglected, i.e., we can first solve P3 without constraints (Equation 28) and then using (Equation 28) to examine the correctness of the solution. So P3 can be efficiently solved by using the Newton Method, which is a method suitable for the convex optimization problem without constraints [22]. In some cases, we can obtain the close form optimal solution $\left(\lambda^{*}, \mu^{*}\right)$ to P3.

Case 1. $\lambda=0$. If $\lambda=0$, then $g(\lambda, \mu)$ degenerates into $g(0, \mu)$. By solving $\frac{\partial g(0, \mu)}{\partial \mu}=0$, we can obtain the optimal $\mu$ :

$$
\mu^{*}=\frac{z}{\ln 2} \frac{\left|\mathcal{L}_{j}\right|}{P 0+\sum_{l \in \mathcal{L}_{j}} b_{j}^{l}} .
$$

Case 2. $\mu=0$. If $\mu=0$, then $g(\lambda, \mu)$ degenerates into $g(\lambda, 0)$. By solving $\frac{\partial g(\lambda, 0)}{\partial \lambda}=0$, we can obtain the optimal $\lambda$ :

$$
\lambda^{*}=\frac{z}{\ln 2} \frac{\left|\mathcal{L}_{j}\right|}{\bar{I}_{j}+\sum_{l \in \mathcal{L}_{j}} c_{j}^{l} b_{j}^{l}}
$$

When the optimal $\left(\lambda^{*}, \mu^{*}\right)$ is obtained, we can use Equation 26 and solve $\frac{\partial L\left(\lambda^{*}, \mu^{*}, \mathbf{p}_{j}\right)}{\partial p_{j}^{l}}=0$ to obtain the optimal $p_{j}^{l *}$. Due to the elimination of $\theta$, we must verify the correctness of the power results. If $p_{j}^{l *} \geq 0$ for all $l \in \mathcal{L}_{j}$, we can conclude that $\mathbf{p}_{j}^{*}$ is the solution to P2. If some elements of $\mathbf{p}_{j}^{*}$ are negative, we must remove these subchannels with the minimum power and solve P3 again until a solution $p_{j}^{l *} \geq 0$ for all $l \in \mathcal{L}_{j}$ is found.

The power optimization procedure is summarized in Algorithm 3. It is difficult to analyze the complexity of Algorithm 3 directly. But the number of iteration times of the Newton Method of Algorithm 3 can be estimated in some extreme cases. In the best cases where the optimal value can be achieved by (Equation 29) or (Equation 30), the iteration time of the Newton Method is one. In the worst case where the optimal value is obtained without 


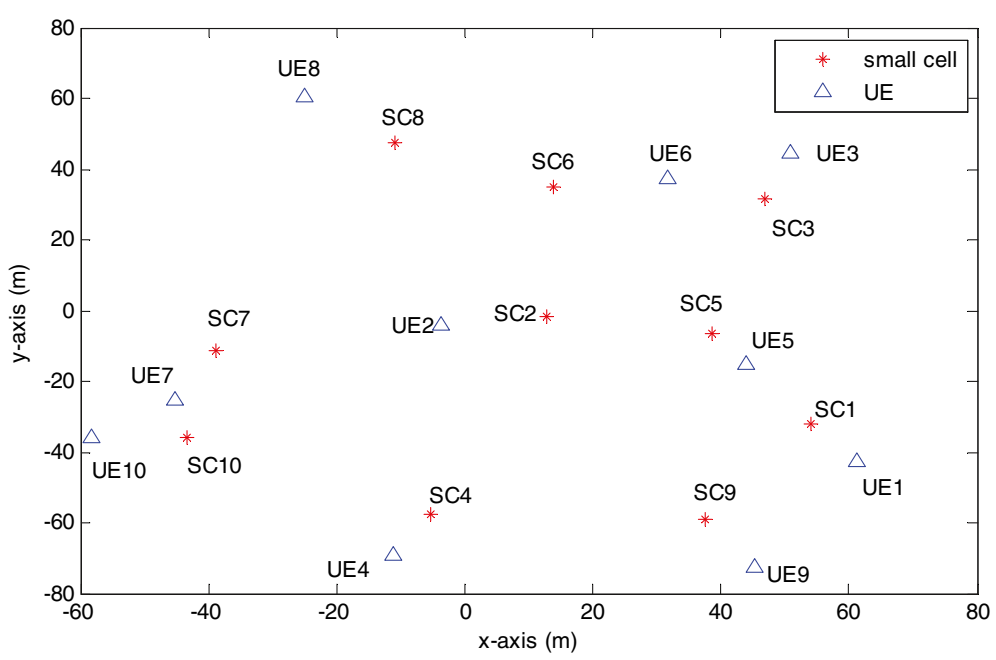

Figure 5 An example of the small cell network topology.

using these close form equations, the maximum number of iterations is bounded by $\frac{g\left(\lambda^{0}, \mu^{0}\right)-g\left(\lambda^{*}, \mu^{*}\right)}{\tau}+6$ [22], where $\lambda^{0}$ and $\mu^{0}$ separately represent the initialized value of $\lambda$ and $\mu$, respectively, and $\tau$ is the maximum reduction value of function $g(\lambda, \mu)$ during the iterations.

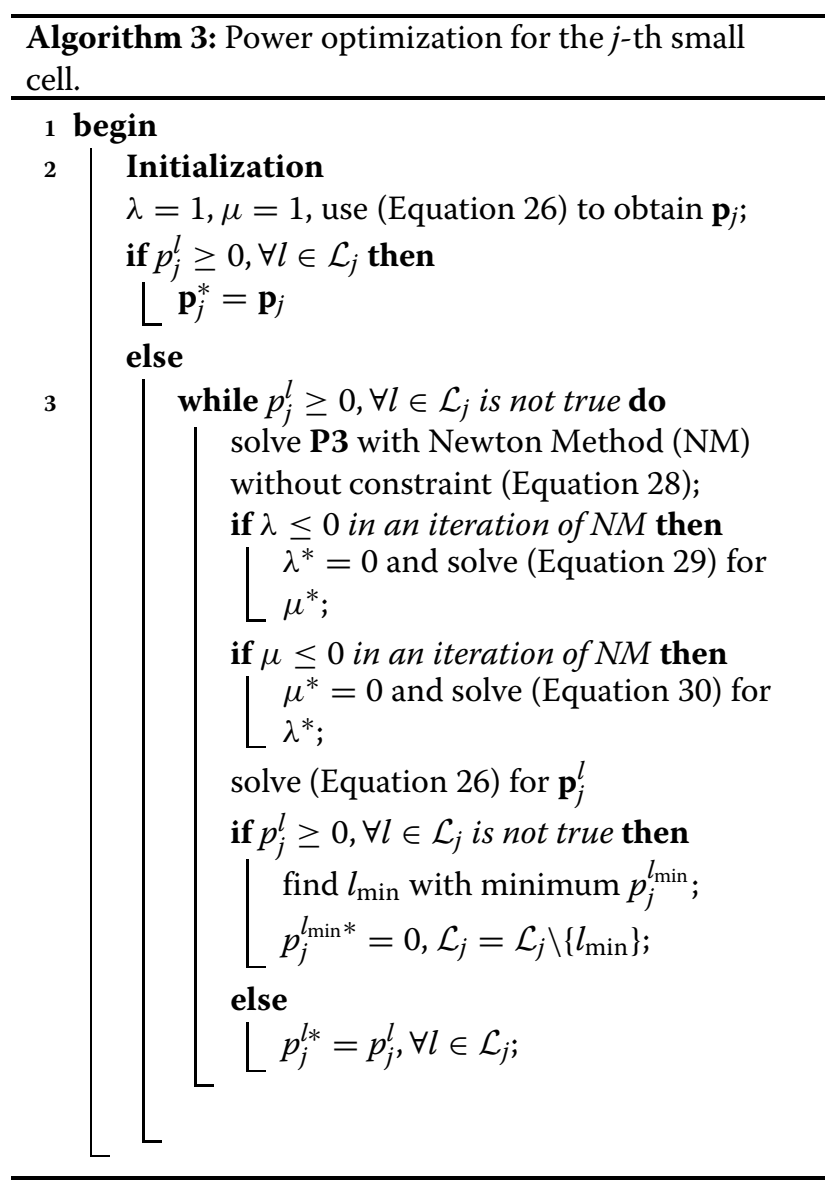

\section{Simulations and discussions}

In this section, system throughput, power consumption, and interference variation are tracked and analyzed during the conduction of RBEBPC. Then, the average performance of the RBEBPC is evaluated. Based on the suggestion of [23], the deployment of small cells is based on the concept of cluster. A small cell cluster consists of a certain number of small cells that are randomly deployed in a certain area. The interference among different small cell clusters can be ignored because the total transmission power of a small cell is small and the distance between clusters is far. For simplicity, in our simulation, we use the square as the area of cluster and focus on the performance of single small cell cluster. The small cells are uniformly distributed in the square with side length $d$ meters. UEs are uniformly distributed in the circles whose center is the serving small cells and radius equals $R$ meters. Figure 5 shows a distribution example of small cells and UEs with parameters $d=120, N=M=10$. Besides, a minimum distance $r$ is set between the small cell and the served UE.

Table 1 Simulation parameters

\begin{tabular}{lc}
\hline Parameters & Value \\
\hline$N$ & 10 \\
$M$ & 10 \\
$F 2(\mathrm{MHz})$ & $1.25 / 5$ \\
$L$ & $6 / 25$ \\
$d(\mathrm{~m})$ & 100 to 160 \\
$(R(\mathrm{~m}), r(\mathrm{~m}))$ & $(15,10),(20,15),(25,20),(20,10)$ \\
System carrier frequency $(\mathrm{GHz})$ & 3.5 \\
Noise power density $(\mathrm{dBm} / \mathrm{Hz})$ & -174 \\
$P 0(\mathrm{dBm})$ & $14 / 20$ \\
Path loss model & Table A.2.1.1.2-3 in $[23]$ \\
\hline
\end{tabular}




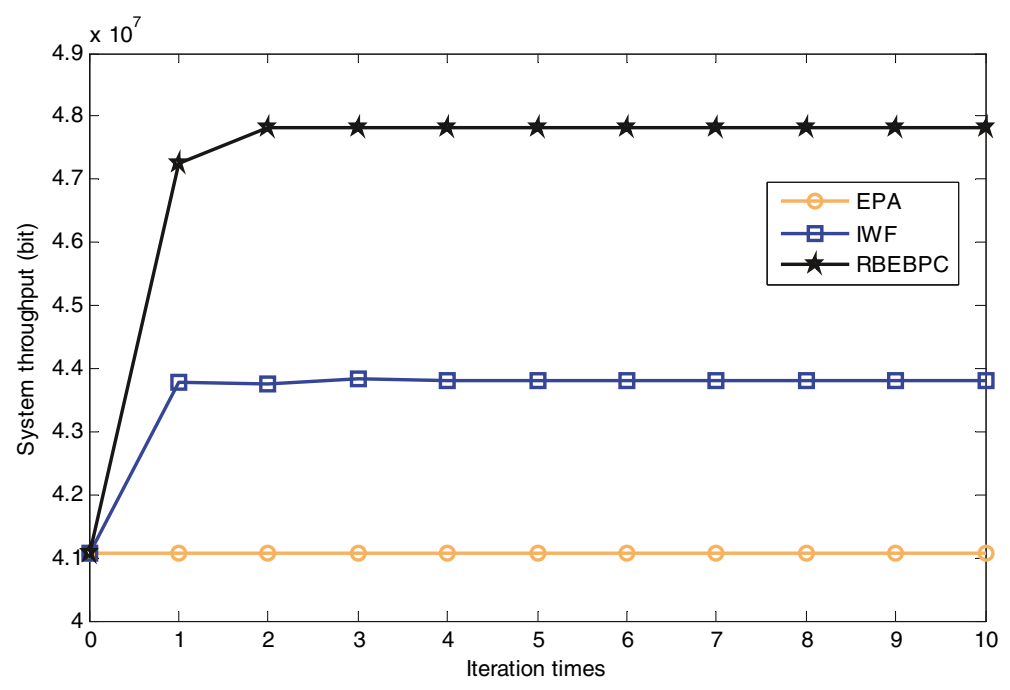

Figure 6 Track of the system throughput after each iteration.

The simulation parameters are summarized in Table 1. As a comparison, the performance of EPA and IWF is also tracked.

\section{Track of RBEBPC}

In this subsection, the system performance of RBEBPC based on the topology of Figure 5 is analyzed. For convenience, the value of $K$ in Algorithm 1 equals 10 and the number of sub-channels is $L=6$. The maximum power of each small cell is $14 \mathrm{dBm}$. The distance pair $(R, r)$ equals $(20,10)$ and bandwidth F2 equals 1.25 M.

Figure 6 shows the system throughput variations of EPA, IWF, and RBEBPC. The initial points of Figure 6 are the system throughput of EPA. RBEBPC outperforms IWF, and IWF outperforms EPA. IWF arrives at the slight fluctuation state after the second iteration and RBEBPC arrives at the stable state after the third iteration.

The resource block exclusion results of the first round and the second round are shown in Tables 2 and 3 separately. After the second round $(k \geq 3)$, no merge or split operation of the resource blocks happens. From the exclusion results, we can see that the merge or split operation happens frequently in the first or second iteration. So the value of $K$ in Algorithm 1 can be set as a small positive integer. From Tables 2 and 3, we can see that coalitions containing two or less players are frequently formed. No grand coalition is formed.

Figure 7 shows the throughput difference between RBEBPC and IWF after each iteration. On the one hand, only small cell 6 , small cell 7 , and small cell 9 have negative throughput growth in RBEBPC compared to IWF. We can see from Table 2 that in the first round, small cell 6 joints the coalitions that has more than two players on all the available sub-channels. Only on sub-channel 4, small cell 6 is the player with advantage, which means that in the following iterations, small cell 6 only occupies the subchannel 4. Small cell 7 is in the same condition as the small cell 6. Due to the interference constraint of Equation 22, small cell 9, will not greedily allocate the power to the sub-channel with the best channel gain. To see this, we can refer to the final power allocation results of IWF and RBEBPC in Tables 4 and 5 separately. From the topology of Figure 5, we can see that the possible coalition player of small cell 9 is small cell 1 and small cell 5 . In the first round of resource block exclusion (Table 2), small cell 9
Table 2 Resource exclusion results, $k=1$

\begin{tabular}{ccc}
\hline $\boldsymbol{l}$ & \multicolumn{1}{c}{$\mathcal{P}^{*}$} & $\boldsymbol{U}$ \\
\hline 1 & $\{1\}\{2\}\{4\}\{5\}\{8\}\{9\}\{3,6\}\{7,10\}$ & $\{1,2,4,5,8,9,3,10\}$ \\
2 & $\{2\}\{4\}\{5\}\{8\}\{1,9\}\{3,6\}\{7,10\}$ & $\{2,4,5,8,1,3,10\}$ \\
3 & $\{1\}\{2\}\{3\}\{4\}\{5\}\{9\}\{10\}\{6,7,8\}$ & $\{1,2,3,4,5,9,10,8\}$ \\
4 & $\{5\}\{7\}\{8\}\{10\}\{1,9\}\{2,4\}\{3,6\}$ & $\{5,7,8,10,1,4,6\}$ \\
5 & $\{4\}\{8\}\{9\}\{1,5\}\{3,6\}\{2\}\{7,10\}$ & $\{4,8,9,1,3,2,10\}$ \\
6 & $\{1\}\{4\}\{5\}\{8\}\{9\}\{10\}\{2,7\}\{3,6\}$ & $\{1,4,5,8,9,10,2,3\}$ \\
\hline
\end{tabular}

Table 3 Resource exclusion results, $k=2$

\begin{tabular}{lcc}
\hline $\boldsymbol{l}$ & $\mathcal{P}^{*}$ & $\boldsymbol{U}$ \\
\hline 1 & $\{1\}\{2\}\{3\}\{4\}\{5\}\{8\}\{9\}\{10\}$ & $\{1,2,3,4,5,8,9,10\}$ \\
2 & $\{1\}\{2\}\{3\}\{4\}\{5\}\{8\}\{10\}$ & $\{1,2,3,4,5,8,10\}$ \\
3 & $\{1\}\{2\}\{3\}\{4\}\{5\}\{8\}\{9\}\{10\}$ & $\{1,2,3,4,5,8,9,10\}$ \\
4 & $\{1\}\{4\}\{5\}\{6\}\{7\}\{8\}\{10\}$ & $\{1,4,5,6,7,8,10\}$ \\
5 & $\{1\}\{2\}\{3\}\{4\}\{8\}\{9\}\{10\}$ & $\{1,2,3,4,8,9,10\}$ \\
6 & $\{1\}\{3\}\{4\}\{5\}\{9\}\{10\}\{2,8\}$ & $\{1,3,4,5,9,10,8\}$ \\
\hline
\end{tabular}




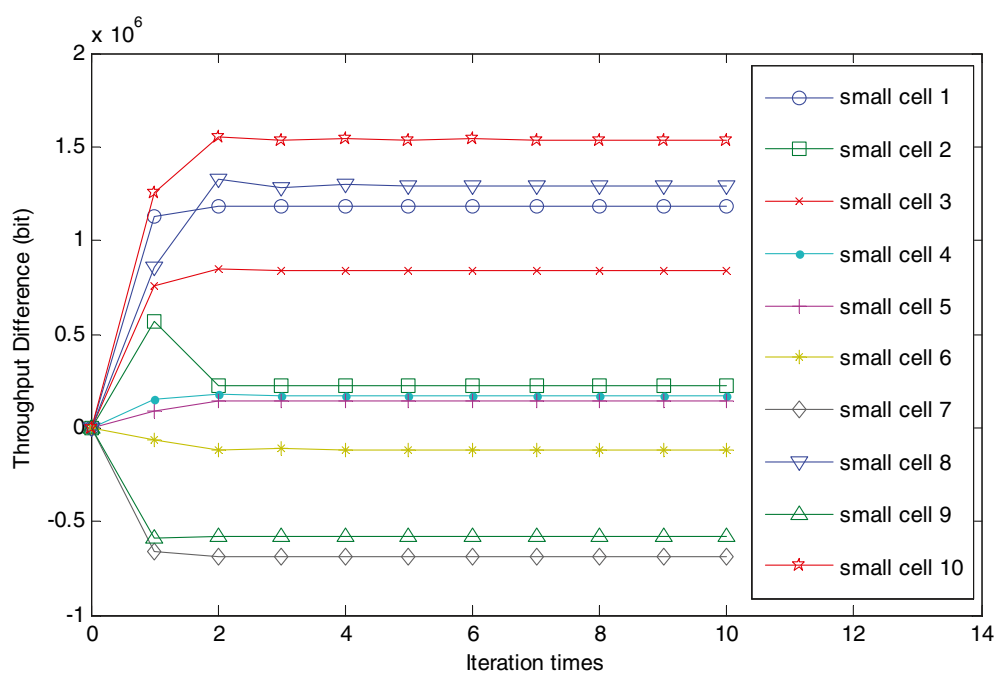

Figure 7 Track of throughput difference after each iteration.

abandons sub-channel 2 and sub-channel 4 compared to IWF. So the abandon of sub-channel and the interference constraint lead to the throughput reduction of small cell 9 . On the other hand, the rest seven small cells benefit from RBEBPC compared to IWF.

Compare Table 4 to Table 5 and we can see that RBEBPC is energy-saving, i.e., not all small cells transmit with the total available power. The small cells can be divided into three groups based on the two tables. The first group (including small cells 2, 6, 7, and 9) consists of the small cells whose total transmit power and available resource blocks vary greatly in RBEBPC compared to IWF. The small cells belong to the first group follow the constraint (Equation 22) and transmit without full power. The second group (including small cell 3) consists of the small cells that use all the available power but different resource blocks compared to IWF. Small cells in the second group are influenced by the resource block exclusion operation. The disadvantage resource blocks are excluded. The third group (including small cell s1, 4, 5, 8, and 10) consists of the small cells that only have different power on all the sub-channels compared with IWF. In the third group of small cells, the power allocation difference among RBEBPC and IWF comes from the other small cells because all the small cells in the system are influenced by the interference. The power allocation variation of the other two groups leads to the power allocation result of the third group.

Figure 8 shows the system total interference under different iterations. The total system interference decreases in both IWF and RBEBPC. At the stable state, RBEBPC further reduces the interference by $56 \%$. Compared to Figure 6, the reduction of interference transforms into the growth of system throughput.

Table 4 Power allocation results of IWF (Watt)

\begin{tabular}{llllllll}
\hline & $\boldsymbol{I = 1}$ & $\boldsymbol{I = 2}$ & $\boldsymbol{I}=\mathbf{3}$ & $\boldsymbol{I = 4}$ & $\boldsymbol{I = 5}$ & $\boldsymbol{I = 6}$ & Total \\
\hline SC1 & 0.0043 & 0.0041 & 0.0046 & 0.0045 & 0.0042 & 0.0034 & 0.0251 \\
\hline SC2 & 0.0025 & 0.0065 & 0.0077 & 0 & 0.0034 & 0.0050 & 0.0251 \\
\hline SC3 & 0.0044 & 0.0033 & 0.0046 & 0.0037 & 0.0047 & 0.0044 & 0.0251 \\
\hline SC4 & 0.0042 & 0.0040 & 0.0041 & 0.0043 & 0.0043 & 0.0042 & 0.0251 \\
\hline SC5 & 0.0049 & 0.0049 & 0.0049 & 0.0052 & 0 & 0.0052 & 0.0251 \\
\hline SC6 & 0.0041 & 0.0092 & 0.0094 & 0.0025 & 0 & 0.0251 \\
\hline SC7 & 0.0041 & 0.0131 & 0 & 0.0080 & 0 & 0.0251 \\
\hline SC8 & 0.0050 & 0.0003 & 0.0050 & 0.0050 & 0.0048 & 0.0050 & 0.0251 \\
\hline SC9 & 0.0039 & 0.0043 & 0.0047 & 0.0029 & 0.0049 & 0.0044 & 0.0251 \\
\hline SC10 & 0.0043 & 0.0036 & 0.0044 & 0.0043 & 0.0043 & 0.0044 & 0.0251 \\
\hline
\end{tabular}


Table 5 Power Allocation Results of RBEBPC (Watt)

\begin{tabular}{|c|c|c|c|c|c|c|c|}
\hline & $I=1$ & $I=2$ & $I=3$ & $I=4$ & $I=5$ & $I=6$ & Total \\
\hline $\mathrm{SC} 1$ & 0.0041 & 0.0044 & 0.0043 & 0.0045 & 0.0041 & 0.0038 & 0.0251 \\
\hline $\mathrm{SC2}$ & 0.0026 & 0.0046 & 0.0028 & 0 & 0.0124 & 0 & 0.0224 \\
\hline $\mathrm{SC} 3$ & 0.0055 & 0.0026 & 0.0056 & 0 & 0.0069 & 0.0046 & 0.0251 \\
\hline $\mathrm{SC} 4$ & 0.0042 & 0.0040 & 0.0041 & 0.0043 & 0.0042 & 0.0043 & 0.0251 \\
\hline SC5 & 0.0032 & 0.0046 & 0.0077 & 0.0052 & 0 & 0.0044 & 0.0251 \\
\hline SC6 & 0 & 0 & 0 & 0.0042 & 0 & 0 & 0.0042 \\
\hline SC7 & 0 & 0 & 0 & 0.0042 & 0 & 0 & 0.0042 \\
\hline $\mathrm{SC} 8$ & 0.0042 & 0.003 & 0.0045 & 0.0040 & 0.0040 & 0.0047 & 0.0251 \\
\hline SC9 & 0.0110 & 0 & 0.0062 & 0 & 0.0042 & 0.0021 & 0.02344 \\
\hline $\mathrm{SC} 10$ & 0.0042 & 0.0041 & 0.0042 & 0.0042 & 0.0041 & 0.0042 & 0.0251 \\
\hline
\end{tabular}

\section{Average performance of RBEBPC}

In this subsection, we will provide the average performance of RBEBPC. The used system parameters are $P 0=$ $20 \mathrm{dBm}, F 2=5 \mathrm{MHz}, L=25$. The proposed overlapping coalition formation (OCF) scheme in [18] is also simulated as the comparison scheme. Note that when we conduct the OCF, the power limitation of coalition formation is removed, i.e., all the small cells in the simulation areas have the potentials to form coalition.

Figure 9 shows the average system throughput of different values of $K$ in Algorithm 2. The difference of average throughput can be neglected when $K$ is bigger than 2. So in practice, the value of $K$ can be set as a small positive number such as 2 or 3 .

Figure 10 shows the average system throughput under different simulation areas. For each scheme, the average system throughput increases as the simulation area becomes bigger. The proposed RBEBPC scheme outperforms IWF by about $15 \%$ in dense case $(d=100)$ and by about $10 \%$ in sparse case $(d=160)$. IWF outperforms the OCF scheme. First, in OCF, the small cells are cooperatively scheduled rather than greedily transmit to achieve the maximum throughput. Second, no power control is used during the transmission in OCF. The gains of RBEBPC, IWF, and OCF over the EPA become weaker as $d$ increases because the interference is not a key factor that influence the system throughput in the sparse scenario.

Figure 11 shows the cumulative distribution function (CDF) of the average system throughput. From the distribution, we can see that under these parameters, the OCF scheme improves the system throughput of EPA by about $30 \mathrm{Mbit}$, IWF improves the system throughput of OCF by about $20 \mathrm{Mbit}$ and RBEBPC improves the system throughput of IWF by about $20 \mathrm{Mbit}$, which indicates

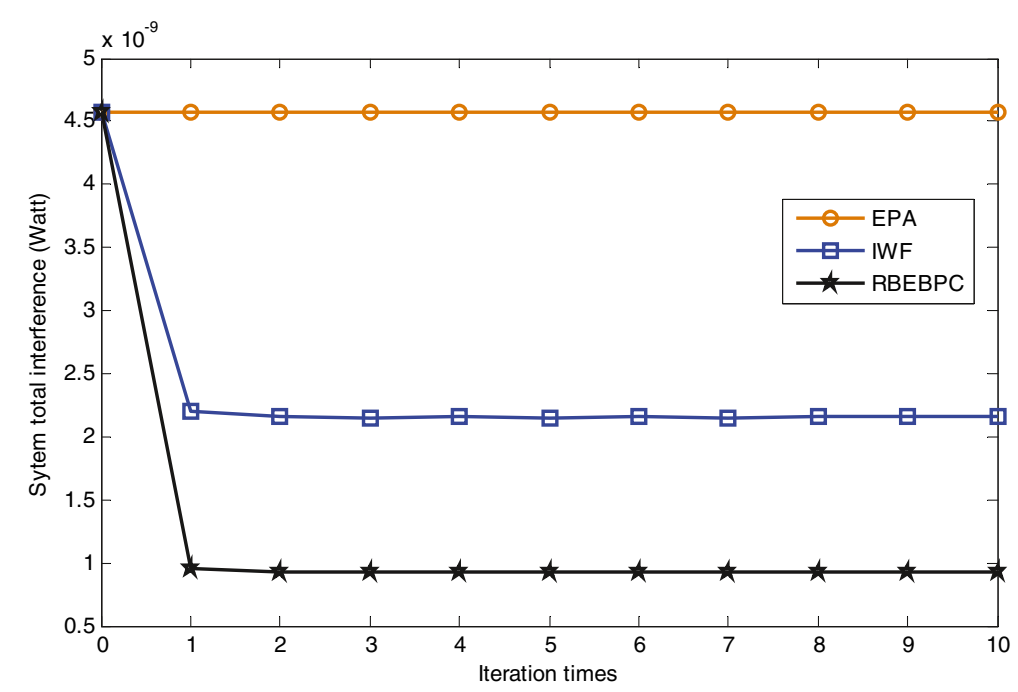

Figure 8 Track of system interference after each iteration. 


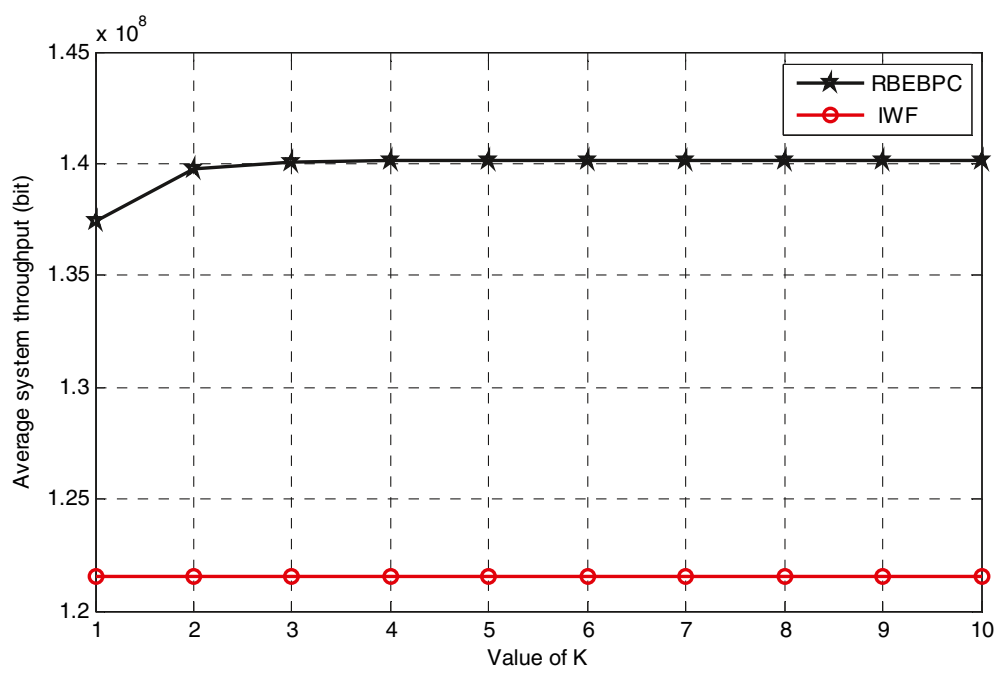

Figure 9 Average system throughput of RBEBPC under different values of $K,(R, r)=(20,10), d=100$.

that $\mathrm{RBEBPC}$ is an effective method to improve the system throughput.

Figure 12 shows the CDF of small cell throughput growth in RBEBPC over the IWF scheme. About $36 \%$ of small cell suffers the loss of throughput (point A). Half of the throughput-decreasing small cells suffer a growth rate of $-24 \%$ (point B). In the rest throughput-increasing small cells, half of them have a growth rate of $22 \%$ (point C). The extreme small cells, whose growth rate is more than $100 \%$ (point D), occupy about $5 \%$ of the total small cells. So the benefit of small cells in RBEBPC is bigger than the loss of the victim small cells.

Figure 13 shows the average system throughput of different link qualities. We consider three cases: the strong link, the moderate link, and the weak link. The elements of the triples represents the growth rate of IWF, OCF, and RBEBPC to the EPA separately. For all the schemes, the average system throughput grows when the link quality become stronger. The growth rates over EPA scheme become bigger when the link quality is weak because in this scenario, the interference has a stronger effect on the system throughput and the mitigation of interference achieves more significant payback in throughput.

Figure 14 shows the average system power consumption of different simulation areas. The IWF/EPA scheme will always use all the available power, while in RBEBPC, not all power is used. Compared with IWF/EPA, about

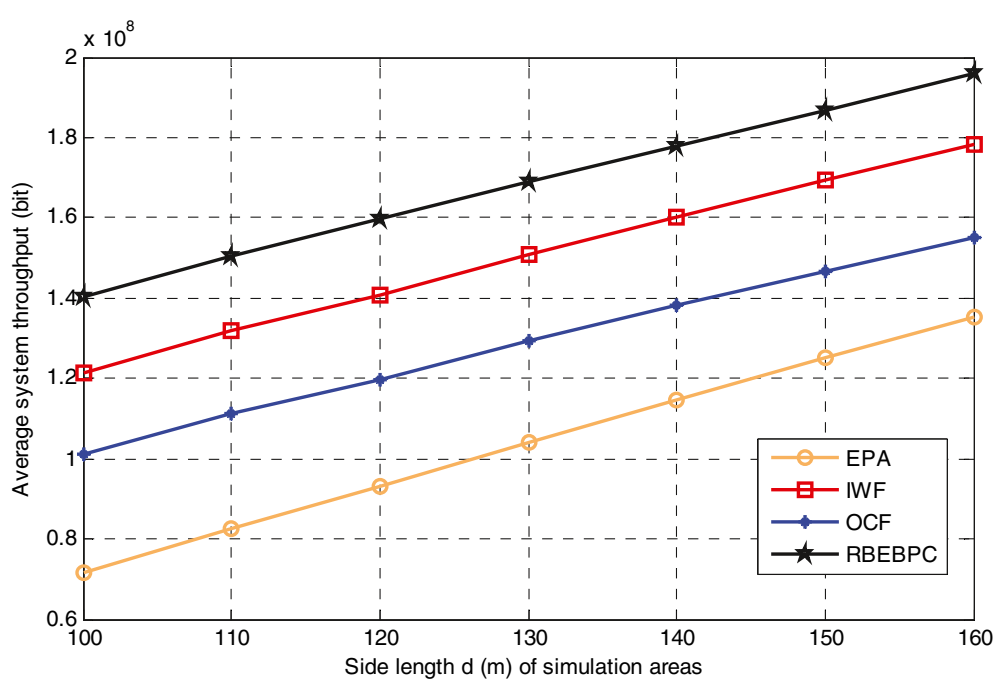

Figure 10 Average system throughput under different $d,(R, r)=(20,10), K=10$. 


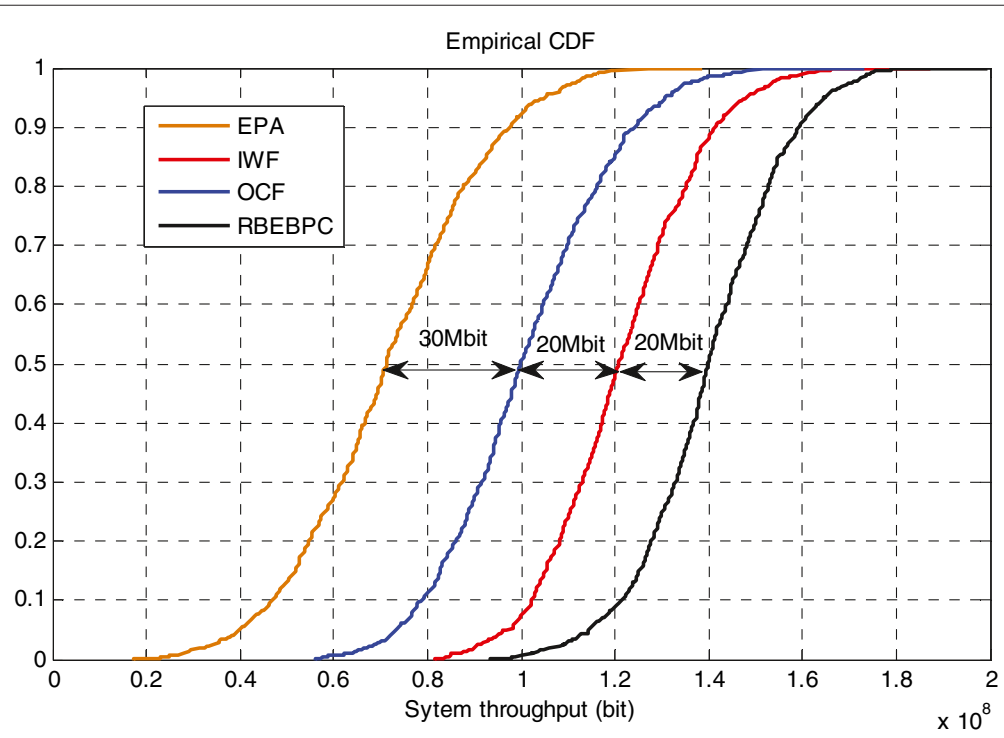

Figure 11 CDF of system throughput, $d=100,(R, r)=(20,10), K=10$.

$13 \%$ power is saved in dense scenario $(d=100)$. In spare deployment scenario, the small cells are prone to use more power. If the deployment of small cells is sparse enough, the interference among small cells will be neglected and the constraint in Equation 22 can be ignored and RBEBPC degenerates into IWF which will consume all the available power.

\section{Conclusions}

This paper focuses on the system throughput improvement via cooperative power control in OFDMA small cell networks. Based on the system resource structure, a cooperative power control scheme named as RBEBPC is proposed. Two steps are iteratively conducted in RBEBPC. First, the small cells play $L$ independent coalition formation games to cooperatively determine the exclusion of resource blocks. Interference constraints are calculated based on the exclusion results for each small cell. Second, each small cell solves a modified throughput maximization problem to determine the power level on each sub-channel. By following the interference constraints, the system total interference is non-increasing and part of the small cells transmit without full power. Simulation results show that both system throughput and energy consumption are improved in RBEBPC compared to the traditional IWF scheme. However, the conduction of RBEBPC relies

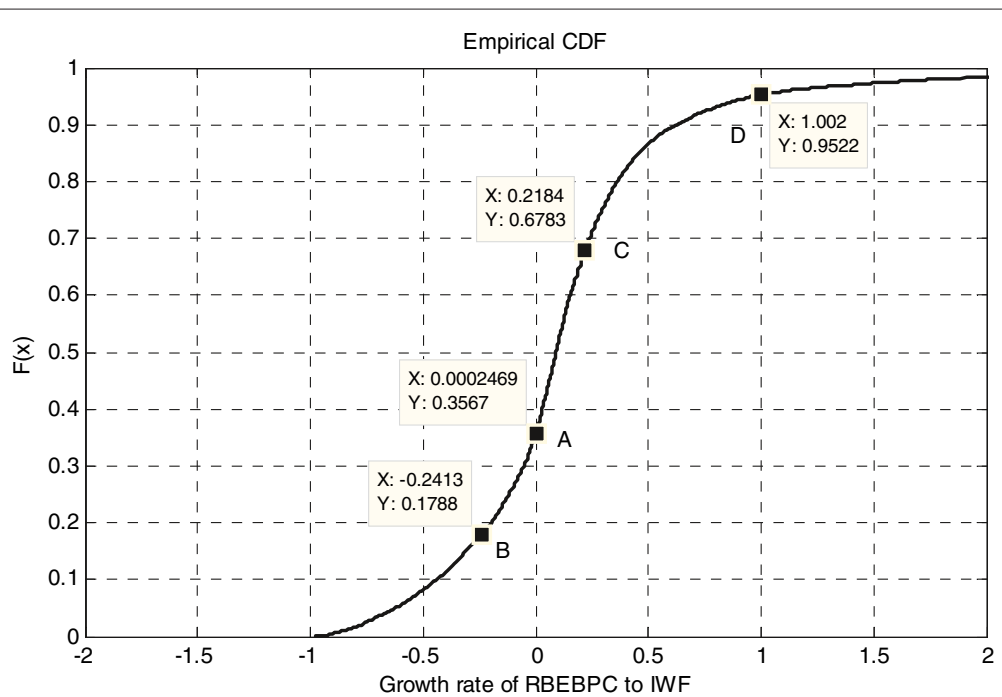

Figure $12 \mathrm{CDF}$ of throughput growth rate, $d=100,(R, r)=(20,10), K=10$. 


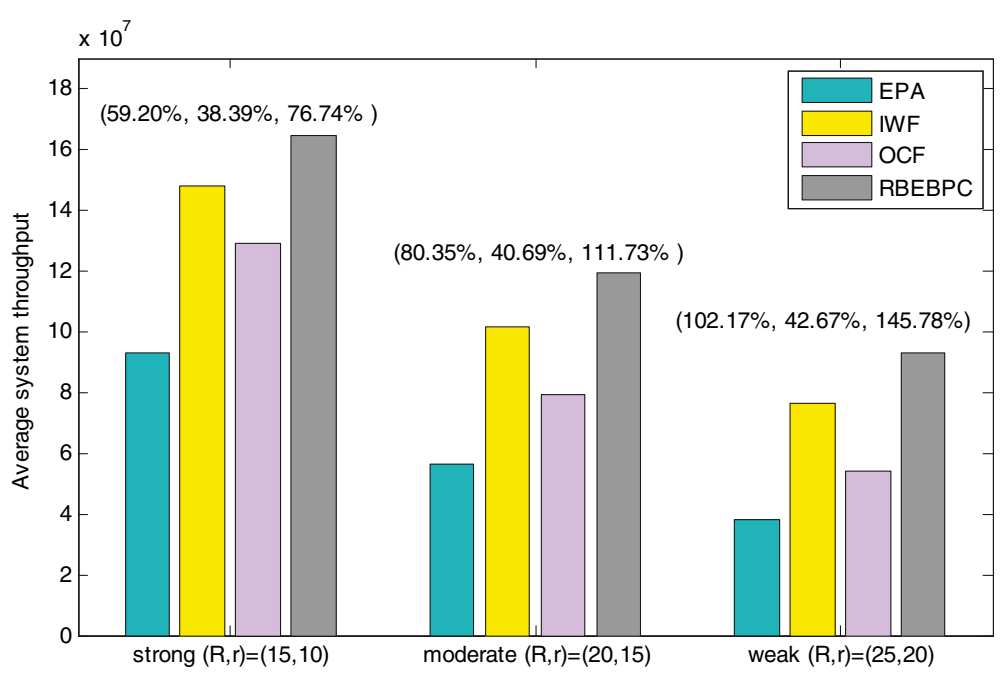

Figure 13 Average system throughput under different link quality, $d=100, K=10$.

on the backhaul link ability between the small cell and the macro cell. The performance improvement is based on the performance sacrifice of partial small cells, which may lead to the unfairness of the network. Both signaling overhead optimization and small cell fairness will be considered in our future study.

\section{Appendix}

\section{Proof of Property 2}

Proof. Consider two coalitions, $\mathcal{E}_{1} \subseteq \mathcal{N}_{l}, \mathcal{E}_{2} \subseteq \mathcal{N}_{l}$ and $\mathcal{E}_{1} \cap \mathcal{E}_{2}=\emptyset$, which satisfy $a$ ) slightly interference with each other such that $\tilde{R}\left(\mathcal{E}_{1} \cup \mathcal{E}_{2}\right) \leq R\left(\mathcal{E}_{1} \cup \mathcal{E}_{2}\right)=R\left(\mathcal{E}_{1}\right)+R\left(\mathcal{E}_{2}\right)$ and $b$ ) strong interference within each coalition such that $\tilde{R}\left(\mathcal{E}_{1}\right)>R\left(\mathcal{E}_{1}\right)$ and $\tilde{R}\left(\mathcal{E}_{2}\right)>R\left(\mathcal{E}_{2}\right)$. In this case, $v\left(\mathcal{E}_{1} \cup\right.$
$\left.\mathcal{E}_{2}\right)=0<\tilde{R}\left(\mathcal{E}_{1}\right)+\tilde{R}\left(\mathcal{E}_{2}\right)=v\left(\mathcal{E}_{1}\right)+v\left(\mathcal{E}_{2}\right)$, which means game $\mathcal{G}^{l}$ is not supperadditive.

\section{Proof of Property 3}

Proof. The $\mathbb{D}_{h p}$ stability of partition structure obtained by Algorithm 2 follows by the termination condition of Algorithm 2. The necessary and sufficient conditions for $\mathbb{D}_{c}$ stability of a partition can be found in [24]. Since the partition structure of $\mathcal{G}^{l}$ is based on location and wireless channel state which are all random variables, these conditions are not always satisfied. But if $\mathbb{D}_{c}$ stable partition of $\mathcal{G}^{l}$ exists, the merge-split rule converges to this partition because the optimal $\mathbb{D}_{c}$ stable partition is the unique outcome of any arbitrary merge-split rule operation [24].

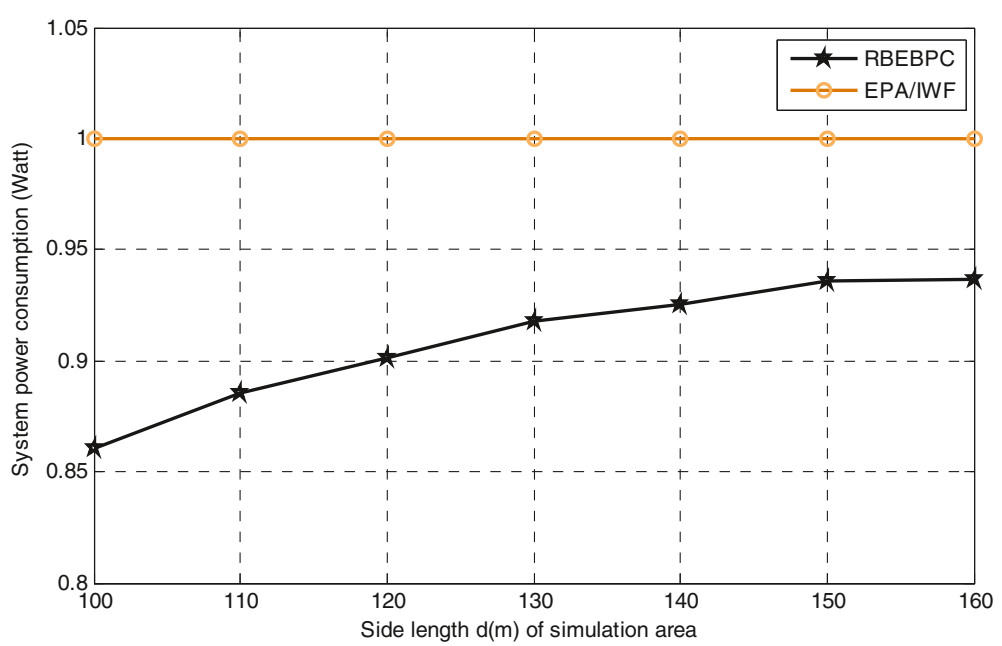

Figure 14 Average system power consumption of different $d,(R, r)=(20,10), K=10$. 


\section{Concavity Proof of P2}

Proof. The domain of $\mathbf{P 2}$ is non-empty because at least the solution $[0,0, \ldots, 0]^{T} \in \mathbb{R}^{\left|\mathcal{L}_{j}\right| \times 1}$ satisfies all the constraints of P2. Since all the constraints of P2 are linear, the domain formed by these constraints is a convex set. The Hessian of the target function is as follows:

$$
\left[\begin{array}{lllll}
\delta_{j}^{1} & & & & \\
& \delta_{j}^{2} & & & \\
& & & & \\
& & & & \\
& & \delta_{j}^{l} & & \\
& & & \ddots & \\
& & & & \delta_{j}^{\left|\mathcal{L}_{j}\right|}
\end{array}\right] \in \mathbb{R}^{\left|\mathcal{L}_{j}\right| \times\left|\mathcal{L}_{j}\right|},
$$

where $\delta_{j}^{l}=\frac{W}{L \ln 2} \frac{-1}{\left(p_{j}^{l}+\frac{\sigma^{2}+I_{j}^{l}}{G_{j j}^{l}}\right)^{2}}, \forall l \in \mathcal{L}_{j}$. Note that when we calculate the Hessian, the interference on each subchannel is processed as a constant. Since the Hessian of the target function of P2 is a diagonal matrix and each element in the diagonal is negative, the Hessian is negative semidefinite. Based on the second order conditions [22], we conclude that the target function of $\mathbf{P 2}$ is a concave function. So P2 is a concave optimization problem.

\section{Competing interests}

The authors declare that they have no competing interests.

\section{Acknowledgements}

This work was supported by the 'Radio Resource Management for Small Cell Deployment' Project of DOCOMO Beijing Communications Laboratories Co. Ltd, the National Natural Science Foundation of China (Grant No.61471060) and the Funds for Creative Research Groups of China (Grant No.61421061).

\section{Author details}

${ }^{1}$ State Key Laboratory of Networking and Switching Technology, Beijing University of Posts and Telecommunications, Xitucheng Road, 100876 Beijing, China. ${ }^{2}$ DOCOMO Beijing Communication Laboratories Co., Ltd.,Kexueyuan South Road, 100190 Beijing, China.

Received: 4 September 2014 Accepted: 18 February 2015

Published online: 11 March 2015

\section{References}

1. Cisco Visual Networking Index: Global Mobile Data Traffic Forecast Update, 2011-2016. http://www.cisco.com/en/US/solutions/collateral/ ns341/ns525/ns537/ns705/ns827/white_paper_c11-520862.html

2. I Hwang, B Song, SS Soliman, A holistic view on hyper-dense heterogeneous and small cell networks. IEEE Commun. Mag. 51, 20-27 (2013)

3. TR 36.932, Scenarios and Requirements for Small Cell Enhancements for E-UTRA and E-UTRAN (Release 12) (2012)

4. MI Kamel, KMF Elsayed, in Global Telecommunications Conference. Performance evaluation of a coordinated time-domain elCIC framework based on ABSF in heterogeneous LTE-advanced networks (IEEE Piscataway, New Jersey, USA, 2012), pp. 5326-5331

5. L Jiang, M Lei, in Personal Indoor and Mobile Radio Communications. Resource allocation for elCIC scheme in heterogeneous networks (IEEE Piscataway, New Jersey, USA, 2012), pp. 448-453

6. J Oh, Y Han, in Personal Indoor and Mobile Radio Communications. Cell selection for range expansion with almost blank subframe in heterogeneous networks (IEEE Piscataway, New Jersey, USA, 2012), pp. 653-657

7. J Pang, J Wang, D Wang, G Shen, Q Jiang, J Liu, in Wireless Communications and Networking Conference. Optimized time-domain resource partitioning for enhanced inter-cell interference coordination in heterogeneous networks (IEEE Piscataway, New Jersey, USA, 2012), pp. 1613-1617

8. S Uygungelen, G Auer, Z Bharucha, in Vehicular Technology Conference. Graph-based dynamic frequency reuse in femtocell networks (IEEE Piscataway, New Jersey, USA, 2011), pp. 1-6

9. W Noh, W Shin, C Shin, K Jang, Choi H-h, in Wireless Communications and Networking Conference. Distributed frequency resource control for intercell interference control in heterogeneous networks (IEEE Piscataway, New Jersey, USA, 2012), pp. 1794-1799

10. T Nakamura, S Nagata, A Benjebbour, Y Kishiyamaand, T Hai, S Xiaodong, $Y$ Ning, L Nan, Trends in small cell enhancements in LTE advanced. IEEE Commun. Mag. 51, 98-105 (2013)

11. P Li, Y Zhu, in Personal Indoor and Mobile Radio Communications. Price-based power control of femtocell networks: a Stackelberg game approach (IEEE Piscataway, New Jersey, USA, 2012), pp. 1185-1191

12. S Guruacharya, D Niyato, DI Kim, E Hossain, Hierarchical competition for downlink power allocation in OFDMA femtocell networks. IEEE Trans. Wireless Comm. 12, 1543-1553 (2013)

13. L Giupponi, C Ibars, in Personal Indoor and Mobile Radio Communications. Distributed interference control in OFDMA-based femtocells (IEEE Piscataway, New Jersey, USA, 2010), pp. 1201-1206

14. L Venturino, N Prasad, X Wang, Coordinated scheduling and power allocation in downlink multicell OFDMA networks. IEEE Trans. Veh. Tech. 58, 2835-2848 (2009)

15. J Kimm, D-H Cho, A joint power and subchannel allocation scheme maximizing system capacity in indoor dense mobile communication systems. IEEE Trans. Veh. Tech. 59, 4340-4353 (2010)

16. KW Shum, K-K Leung, CW Sung, Convergence of iterative waterfilling algorithm for Gaussian interference channels. IEEE J. Sel. Area Comm. 25 1091-1100 (2007)

17. F Pantisano, M Bennis, W Saad, R Verdone, M Latva-aho, in Wireless Communications and Networking Conference. Coalition formation games for femtocell interference management: a recursive core approach (IEEE Piscataway, New Jersey, USA, 2011), pp. 1161-1166

18. Z Zhang, L Song, Z Han, W Saad, Z Lu, in Wireless Communications and Networking Conference. Overlapping coalition formation games for cooperative interference management in small cell networks (IEEE Piscataway, New Jersey, USA, 2013), pp. 643-648

19. D Tse, P Viswanath, Fundamentals of Wireless Communication. (Cambridge University Press, USA, 2004)

20. CS Ranaweera, PP lannone, KN Oikonomou, KC Reichmann, RK Sinha, Design of cost-optimal passive optical networks for small cell backhaul using installed fibers. IEEE/OSA J. Opt. Commun. Netw. 5, 230-239 (2013)

21. W Saad, Z Han, M Debbah, A Hjorungnes, T Basar, Coalitional game theory for communication networks. IEEE Signal Process. Mag. 26, 77-97 (2009)

22. S Boyd, L Vandenberghe, Convex Optimization. (Cambridge University Press, USA, 2004)

23. 3GPP, TR 36.814, Further advancements for E-UTRA physical layer aspects (Release 9) (2010)

24. KR Apt, T Radzik, in arXiv:CS/0605132v1 [Cs.GT]. Stable partitions in coalitional games (New York, USA, May, 2006) 